\title{
Targeted and contextual redescription set exploration
}

\author{
Matej Mihelčićć,2 ${ }^{1, T o m i s l a v ~ S ̌ m u c ~}{ }^{1}$
}

Received: 31 March 2017 / Accepted: 25 June 2018 / Published online: 5 July 2018

(c) The Author(s) 2018

\begin{abstract}
One important problem occurring in redescription mining is a very large number of produced redescriptions. This makes analyses time consuming and generally difficult. We present the targeted and contextual redescription set exploration, realized through the tool InterSet. The main purpose of the tool is to derive additional knowledge from the redescription set which allows exploring parts of redescription set of interest and examining redescriptions individually or in the broader context, with the aim of increasing overall understandability. InterSet allows relating, grouping redescriptions, observing distributions of various redescription properties and selecting the appropriate subsets for further, detailed study. This allows gaining knowledge about the underlying data, help in forming, understanding, supporting research hypothesis or assists in understanding one or more redescriptions of interest. The tool provides three different, fully connected interaction modes based on: (1) similarity of entity occurrence in redescription support sets, (2) attribute co-occurrence in redescriptions and (3) redescription quality measures. Additionally, it allows exploration of relations between different redescriptions by creating a graph visualization that includes the top $k$-shortest paths containing selected redescriptions. On the individual redescription level, it allows studying value distributions of described entities, for a given set of attributes.
\end{abstract}

Keywords Knowledge discovery $\cdot$ Redescription mining $\cdot$ Redescription set $\cdot$ k-paths Interactive exploration $\cdot$ Self organising map $\cdot$ Heatmap $\cdot$ Crossfilter

Editors: Toon Calders and Michelangelo Ceci..

Electronic supplementary material The online version of this article (https://doi.org/10.1007/s10994-0185738-9) contains supplementary material, which is available to authorized users.

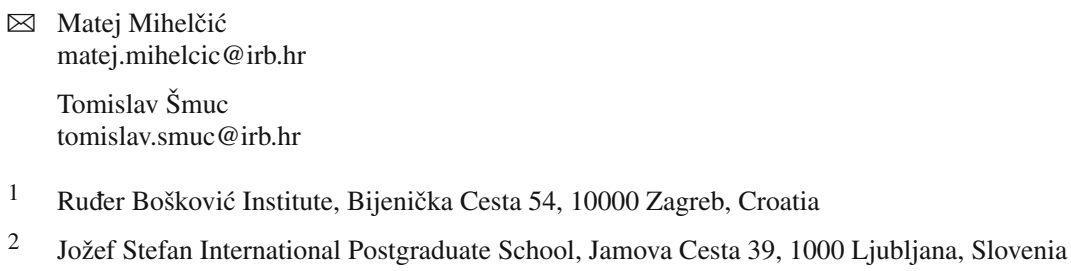




\section{Introduction}

Redescription mining (Ramakrishnan et al. 2004) is a descriptive data mining task focused at finding redescriptions of different subsets of entities from the available data. Redescription consists of two or more descriptions (logical formulas called queries) depicting same, or very similar subset of entities (which constitute redescription support set), by using information from different sources, describing different aspects of entities, describing entities at different points in time etc. The intuition behind redescription mining is that queries describing similar sets of entities provide information about the shared properties of these entities.

Various redescription mining algorithms (Ramakrishnan et al. 2004; Zaki and Ramakrishnan 2005; Parida and Ramakrishnan 2005; Gallo et al. 2008; Galbrun and Miettinen 2012b; Mihelčić et al. 2015; Zinchenko 2014) are capable of producing potentially large sets of redescriptions often containing similar or redundant redescriptions. Thus, it is important to develop tools for efficient redescription set exploration. The existing approach, implemented in the interactive redescription mining environment Siren (Galbrun and Miettinen 2012b, 2016), focuses on redescription set exploration based on analysis of individual redescriptions.

The main novelty of our approach is that it uses information derived from the redescription set as a whole (clusters of entities based on redescription supports, attribute co-occurrences and frequencies of attributes in redescriptions, statistics about quality properties of redescriptions) to allow performing contextual and targeted redescription set exploration. Targeted redescription set exploration allows placing immediate focus on a subset of redescriptions directly linked to the research hypothesis, while contextual exploration allows exploring (dis)similar redescriptions to one or more observed redescriptions of interest and finding relations between them.

Exploration can be performed based on any combination of the following properties: entities described by different redescriptions, attributes used to describe these entities and different redescription quality criteria. Relations between redescriptions contained in a small sequence, with respect to described entities, can be determined and efficiently visualized through the redescription relation graph. Relating similar redescriptions (with respect to described entities) discovers the close neighbourhood of redescriptions in question, providing the extended (but very focused) knowledge to the domain experts. On the other hand, relating completely dissimilar sets of entities is also an interesting research topic, studied in more detail in a related research task called storytelling (Kumar et al. 2008). Disjoint sets of entities can be related by creating a sequence of redescriptions such that starting from the first redescription (redescribing the first set of entities), every newly discovered redescription describes similar subset of entities as its predecessor and its successor sharing one query with these redescriptions. The final redescription in the sequence redescribes the second set of entities. By observing this sequence, users can obtain information about apparently unrelated redescriptions (redescribing disjoint sets of entities). Storytelling was applied on three use cases: relating words in English language, relating different gene sets and relating PubMed abstracts. When relating completely dissimilar redescriptions, our approach finds such, but also more relaxed sequences of redescriptions since it does not require that every consecutive pair of redescriptions shares a query (existing highly connected stories would be discovered). For a given sequence of redescriptions, our approach allows displaying $k$ sequences as top $k$ shortest graph paths where each path contains redescriptions with the highest possible overlap with their predecessor and successor extending the initially selected sequence of redescriptions. All initially selected redescriptions are contained in the produced paths in the selection order. 
Table 1 Example redescription $R=\left(q_{1}, q_{2}\right)$

$\begin{array}{ll}q_{1}: & \text { SymponCompGeom } \wedge \text { CCCG } \\ q_{2}: & \text { Boris Aronov } \vee \text { Godfried T. Toussaint }\end{array}$

This work builds upon and significantly extends the work presented in Mihelčić and Šmuc (2016) by introducing new capabilities of the tool, such as full interaction between different aspects, extended attribute and property exploration aspects and the ability to directly relate a sequence of redescriptions, which is an important but not fully explored line of research. Motivation, explanation and argumentation of proposed visualizations are extended and the tool's capabilities are demonstrated over extended set of use cases.

We introduce the notation and provide the illustrative redescription example in Sect. 2. Section 3 contains the related work whereas Sect. 4 motivates the proposed exploration methodology, Sect. 5 describes the data while Sect. 6 provides a detailed motivation and overview of visualizations and functionality of the InterSet tool. ${ }^{1}$ In Sect. 7, we demonstrate the use of the proposed methodology to discover new knowledge on three different datasets, in Sect. 8 we present complexity and execution time analyses of InterSet, while in Sect. 9 we discuss implementation details of the tool. Finally, Sect. 10 contains conclusions and possible directions for future work.

\section{Notation and definitions}

Input to the redescription mining algorithm consists of a set of available entities, denoted with $E$ and a set of attributes which are logically grouped in one or more views, denoted $W_{i}, i \geq 1$. The corresponding set of variables is denoted with $V_{i}, i \geq 1$. All current approaches (including the InterSet) work with maximally two distinct views, thus all available redescriptions describe a set of entities with maximally two different descriptions. Formally, a redescription $R$ is a pair of queries $R=\left(q_{1}, q_{2}\right)$ where each query describes a set of entities by using variables contained in a set of variables belonging to a corresponding view. Queries are logical formulas in which variables or its negations are connected with conjunction and disjunction operators.

We present an example redescription obtained on one of our use case datasets ${ }^{2}$ describing authors of scientific papers. Entities are authors of scientific papers and descriptors are: conferences on which they published a scientific paper (view 1), and authors that co-authored a scientific paper with these authors (view 2). The redescription $R=\left(q_{1}, q_{2}\right)$ contains two queries $q_{1}, q_{2}$ and is defined in Table 1 .

Variables (SympOnCompGeom - Symposium on Computational Geometry, CCCG - Canadian Conference on Computational Geometry) in $q_{1}$ are connected with the conjunction (AND) operator, while variables (Boris Aronov, Godfried T. Toussaint) in $q_{2}$ are connected with the disjunction (OR) operator.

Throughout this work, we use several redescription quality measures: the support, the Jaccard index, the $p$-value, the average element and attribute Jaccard index. The support of a query $q_{i}\left(\operatorname{supp}\left(q_{i}\right)\right)$ is the set of all entities satisfying its condition. The redescription $R=\left(q_{1}, q_{2}\right)$ describes the entity $e$ if $e \in \operatorname{supp}\left(q_{i}\right), \forall i \in\{1,2\}$. All entities described by a redescription compose a redescription support set $\left(\operatorname{supp}(R)=\operatorname{supp}\left(q_{1}\right) \cap \operatorname{supp}\left(q_{2}\right)\right)$.

\footnotetext{
1 http://zel.irb.hr/interset/.

2 Using InterSet tool on all use case data is described in Sect. 7.
} 
Higher similarity among sets of entities described by redescription queries represents higher redescription accuracy. Jaccard index quantifies this similarity and is used as a measure of redescription accuracy. It is defined as:

$$
J(R)=\frac{\left|\operatorname{supp}\left(q_{1}\right) \cap \operatorname{supp}\left(q_{2}\right)\right|}{\left|\operatorname{supp}\left(q_{1}\right) \cup \operatorname{supp}\left(q_{2}\right)\right|}
$$

For a given redescription $R=\left(q_{1}, q_{2}\right), p_{v a l}(R)$ represents a probability of obtaining a set of a size equal to or larger than that of $\operatorname{supp}(R)$, by combining two random queries with marginal probabilities corresponding to the marginal probabilities of queries $q_{1}$ and $q_{2}$. The $p$-value ( $\left.p_{v a l}\right)$, also used by Galbrun and Miettinen (2012a), is computed from the binomial distribution:

$$
p_{\text {val }}(R)=\sum_{n=|\operatorname{supp}(R)|}^{|E|}\left(\begin{array}{c}
|E| \\
n
\end{array}\right)\left(p_{1} \cdot p_{2}\right)^{n} \cdot\left(1-p_{1} \cdot p_{2}\right)^{|E|-n}
$$

$|E|$ denotes the number of entities and $p_{1}=\frac{\left|\operatorname{supp}\left(q_{1}\right)\right|}{|E|}, p_{2}=\frac{\left|\operatorname{supp}\left(q_{2}\right)\right|}{|E|}$ correspond to marginal probabilities of obtaining the query $q_{1}$ and $q_{2}$.

We define $\operatorname{attrs}(R)$ as a set of attributes used in redescription queries and the attribute Jaccard index of two redescriptions as:

$$
\operatorname{att} J\left(R_{1}, R_{2}\right)=\frac{\left|\operatorname{attrs}\left(R_{1}\right) \cap \operatorname{attrs}\left(R_{2}\right)\right|}{\left|\operatorname{attrs}\left(R_{1}\right) \cup \operatorname{attrs}\left(R_{2}\right)\right|}
$$

Given a redescription set $\mathcal{R}$ containing $|\mathcal{R}|$ redescriptions, the average attribute Jaccard index of a redescription $R_{i} \in \mathcal{R}$ is defined as:

$$
\operatorname{AAJ}\left(R_{i}\right)=\frac{\sum_{R_{j} \in \mathcal{R}, j \neq i} \operatorname{att} J\left(R_{i}, R_{j}\right)}{|\mathcal{R}|-1}
$$

By analogy, the entity Jaccard index of two redescriptions is defined as:

$$
\operatorname{elem} J\left(R_{1}, R_{2}\right)=\frac{\left|\operatorname{supp}\left(R_{1}\right) \cap \operatorname{supp}\left(R_{2}\right)\right|}{\left|\operatorname{supp}\left(R_{1}\right) \cup \operatorname{supp}\left(R_{2}\right)\right|}
$$

and the average entity Jaccard index as:

$$
\operatorname{AEJ}\left(R_{i}\right)=\frac{\sum_{R_{j} \in \mathcal{R}, j \neq i} \operatorname{elem} J\left(R_{i}, R_{j}\right)}{|\mathcal{R}|-1}
$$

These measures provide information about the redundancy of a redescription with respect to entities and attributes.

We will use a short notation $a t t_{a}^{b}$ for the expression $a \leq a t t \leq b$ when the attribute contains numerical values and $a t t^{b}$ for the expression $a t t=b$ when the attribute contains categorical values. We define the homogeneity of a cluster of entities $C$ obtained on the redescription selection $\mathcal{R}_{\text {sel }}$ as:

$$
\text { avHom }=\frac{\sum_{R \in \mathcal{G}} J(\operatorname{supp}(R), C)}{|\mathcal{G}|}
$$

where $\mathcal{G}=\left\{R \in \mathcal{R}_{\text {sel }}, \operatorname{supp}(R) \cap C \neq \emptyset\right\}$. 


\section{Related work}

In this work, we focus on developing techniques for targeted and contextual redescription set exploration. To obtain redescriptions, we rely on the algorithms developed in redescription mining, a task introduced by Ramakrishnan et al. (2004). One or more existing redescription mining algorithms (Ramakrishnan et al. 2004; Zaki and Ramakrishnan 2005; Parida and Ramakrishnan 2005; Gallo et al. 2008; Galbrun and Miettinen 2012b; Mihelčić et al. 2015; Zinchenko 2014) can be used to obtain the redescription set that is used as the input to the InterSet tool. These algorithms can be combined into ensembles which reduces the potential algorithmic biases in redescription creation. This can be done by producing a redescription set by each algorithm and storing all produced redescriptions into the input database, used by the InterSet tool.

\subsection{Interactive knowledge discovery}

Standard approach to knowledge discovery usually includes a data scientist who obtains the knowledge by using some data mining or machine learning algorithms and a domain expert whose role is to interpret the obtained knowledge, formed hypothesis etc. It has soon been realized that it would be more efficient to allow domain experts to directly interact with the data mining system. Work from Webb (1996) goes in the direction of closing this gap between domain experts and knowledge acquisition algorithms. Systems like KNIME (Berthold et al. 2009), RapidMiner (Hofmann and Klinkenberg 2013) and ClowdFlows (Kranjc et al. 2012) provide tools to visually create and execute machine learning workflows. Work presented in Boley et al. (2013), Van Leeuwen (2014), and Miettinen (2014) discusses the problem of interactive data mining and pattern selection. Boley et al. (2013) point out the necessity of using interactive approaches in pattern mining because they allow alleviating different data mining specific tasks (such as definition of parameters, choice of a data mining model etc) in the process of pattern acquisition - leaving more time for expert evaluation of patterns. Van Leeuwen (2014) points out the necessity of using interactive approaches due to user and task dependent information that need to be taken into account during pattern mining. He also points out advantages of interactive approaches when performing pattern mining under constrained resources. Miettinen (2014) argues that interactive data mining needs to be used with care, otherwise it can happen that it leads exclusively to those patterns expected by the user.

\subsection{Related exploration and visualization tools}

MIME (Goethals et al. 2011) is a framework for interactive visual pattern mining. Zaki and Phoophakdee (2003) created a tool for exploring, mining and visualizing minimal association rules called MIRAGE. It uses lattice-based visualisation and exploration of minimal association rules. The association rule visualization system for exploratory data analysis (Liu et al. 2012) uses scatter plot to visualize rules from the association rule set. The rules can be explored on the individual level by modifying the rules and observing comparative bar charts prior and post attribute addition or deletion. A user driven and quality oriented visualization for mining association rules (Blanchard et al. 2003) embeds association rules to 3D landscape. It allows users to select rule subsets, navigate among different subsets and to filter them on several interestingness measures by using sliders. Multi level spatial association rules mined by the tool ARES (Appice and Buono 2005) are visualized using graphs. The R programming package called the arules (Michael et al. 2011) allows creating and implementing 
transaction databases. It also offers basic algorithms for finding, analysing and visualizing association rules. The package allows visualizing association rules via scatter plots, two-key plots, 3-D matrices, grouped matrices, and in a graph form. Rule to item association rule 3-D visualization (Chakravarthy and Zhang 2003) allows representing several association rules and their confidence. Andrienko and Andrienko (1999) developed a map based visualization for interactive exploration of spatial data.

The Self Organizing Map (SOM) (Kohonen et al. 2001) is used by Castillo-Rojas et al. (2014) to display the spatial distribution of the entities associated with the association rule on a map. This differs from our approach in which we use the SOM to group similar entities together (based on redescriptions from the redescription set).

The mentioned exploration tools are not appropriate for redescription set exploration, because of redescription specific properties (such as multiple descriptions describing similar sets of entities, stronger equivalence-like relations between attributes etc.). We motivate the chosen visualizations and explain the redescription set exploration process in Sect. 6.

\subsection{Network exploration}

The Apolo (Chau et al. 2011) and the TourViz (Chau et al. 2012) are two tools aimed at exploration of large networks. TourViz enables similar functionality required in our work such as displaying connectivity graph of a set of nodes and displaying close neighbourhood. Our approach does not require visualizing large networks, to present relations between a sequence of redescriptions, since this can be achieved by visualizing a very well constrained subgraph of interest. Allowing nodes to be grouped by some attribute properties, once the graph has been obtained, is an interesting future work direction.

\subsection{Interactive redescription mining and exploration}

Zaki and Ramakrishnan (2005) developed a console based application that allows limited user interventions such as finding attributes describing a given set of entities, finding entities described by a given set of attributes. It also allows placing constraints on entities and attributes, Jaccard index and redescription support to allow interaction with exploration process.

The only approach that allows exploring a set of redescriptions is a tool Siren, developed by Galbrun and Miettinen (2012b, 2016). It is an interactive redescription mining environment allowing creation, exploration and analysis of redescriptions. Siren's design as interactive and any time miner emphasises exploration and analysis of individual redescriptions. A few different available visualizations allow representing various aspects of a redescription. However, they are all limited to the visualization of a single redescription (more information available in Sect. 1 of Online resource 2). Each generated redescription can be expanded by allowing the tool to improve the accuracy by adding new attributes to the redescription queries. Redundant redescriptions can be filtered based on redescription support.

\section{Specifics and motivation for developing InterSet}

Our approach focuses on redescription set exploration, expecting pre-mined set of redescriptions by using one or more redescription mining algorithms. InterSet exploration process utilizes higher level information, obtained from the input redescription set using statisti- 
cal and machine learning approaches to provide additional knowledge not easily obtainable from data. The tool is designed to handle potentially large sets of redescriptions which would otherwise be overly complex and time consuming to understand by manual exploration and inspection. It is expected that large sets can occur by using some redescription mining approaches or ensembles of different redescription mining algorithms when exhaustive investigation is desired (although in most current applications, sets of smaller size were used). Efficient exploration of large sets neccessitates development of various selection and filtering mechanisms aimed at providing users with the desired subsets of redescriptions. The selection and filtering mechanisms available in InterSet allow focusing on interesting subsets of knowledge, including manual selection of desired subset of redescriptions, and eliminating/removing uninteresting redescriptions. The bottom level of exploration is performed by analysing individual redescriptions (inspecting their queries, support and attribute sets as well as various quality measures) and entities through various forms of value distribution analyses.

Our approach provides a framework that allows incorporating ideas from various pattern set mining and pattern selection approaches, designed to create smaller, compressed or userpreferred set of patterns, into redescription set mining and exploration process. Pattern set mining approaches, such as Xin et al. (2005), De Bie et al. (2010), De Raedt et al. (2013), Lam et al. (2014), and Ouali et al. (2017) can be used to construct sets of new redescriptions describing the selected subsets of entities/attributes of interest, whereas pattern selection methods (Xin et al. 2005; Knobbe and Ho 2006; Pei et al. 2007; Ouali et al. 2017; Kalofolias et al. 2016; Mihelčić et al. 2017a) can be used to perform automated and fine-grained selection of redescriptions from a selected subset of redescriptions. Techniques for performing redescription mining with entity/attribute constraints already exist (Zaki and Ramakrishnan 2005; Mihelčić et al. 2017b). Choice of methodology depends on user preferences and the quality of final set of redescriptions depends on predefined redescription and redescription set quality measures. One directly applicable, scalable methodology that can be used for construction of smaller sets of redescriptions, using user-selected subset of redescriptions and allowing definition of user-provided preference information is the generalized redescription set construction procedure (Mihelčić et al. 2017a).

Since the input set to our tool is pre-mined by using some a priori defined, fixed set of parameters (without user intervention and guidance during mining), we believe our approach should be less prone to the problem of discovering only results that the user expects or desires to obtain, which can occur in interactive mining setting (see Miettinen (2014)). Further, since it facilitates the use of ensembles of redescription mining algorithms, redescriptions contained within the input sets should be less prone to various algorithmic biases. Ultimately, using large, exhaustive, sets of redescriptions provides more information to the user, allows domain experts to choose their own path of exploration, enables deriving additional statistical information enhancing exploration capabilities of users (more similar, reinforcing patterns increases the confidence in a result) and allows exploration of various non-trivial associations. Multiple different sets of attributes, associated within a set, can often exist and be relevant for very similar or the same subsets of entities. Choosing one over the other, in a fully automated fashion, is hardly possible without additional user information.

Our approach utilizes web-based, distributed approach, thus it allows easy collaborations of various domain experts working on a common scientific problem. In this setting, researchers potentially interested and exploring distinct parts of discovered redescription set could collaborate and share their results using the provided and computed meta-information and information about other redescriptions to connect their work or put it in a perspective.

To be used in related fields, such as association rule mining, InterSet requires definition of various measures used to assess the quality of rules in a given field. Attribute-based explo- 
Table 2 Algorithm parameters used to create redescription set on a Country dataset (C), DBLP dataset (D) and Phenotype dataset (P). $2 \times 40$ denotes two random initialization with 40 iterations each and $1+R F_{100}$ denotes using a PCT augmented with Random Forest of 100 PCTs (Mihelcic et al. 2018). Additional explanations are available in Sect. 2 of Online Resource 2

\begin{tabular}{lllllllll}
\hline Data & $\min \mathrm{J}$ & $\max \mathrm{p}$ & support & $|\mathcal{R}|$ & $\left|q_{i}\right|$ & Iters. & Lang. & Trees \\
\hline$C$ & 0.5 & 0.01 & $\geq 10$ & 5448 & 8 & 800 & all & 1 \\
$D$ & 0.2 & 0.01 & $\geq 10$ & 3674 & 8 & 120 & all & 1 \\
$P$ & 0.6 & 0.01 & {$[10,650]$} & 6200 & 6 & $2 \times 40$ & Conj, neg & $1+R F_{100}$ \\
\hline
\end{tabular}

ration context might require changes given specificities of a given task (e.g associations in association rule mining are one sided and directed). InterSet can also facilitate understanding of supervised problems (in particular multi-label predictive problems), if redescription mining is performed on input attributes and target attributes as two separate views. In such cases researcher can discover interesting associations between different combinations of input attributes and target variables, which can be also useful for the construction of novel features, feature selection and/or model selection. Thus, we believe it should be interesting for researchers in a wider data mining and machine learning research community.

\section{Use case datasets}

We demonstrate the tool's capabilities on three different use case datasets, the Country dataset (UNCTAD 2014; WorldBank 2014; Gamberger et al. 2014), the DBLP dataset (DBLP 2010; Galbrun 2013) and the Phenotype dataset (Brbić et al. 2016). Country dataset contains 199 world countries, described by general country descriptors (demographic descriptors, unemployment, etc.) as the first view and country trading patterns (with the values of percentage of export (E) and import (I) that a given commodity forms in total country export or import and the information on the ratio of these values (E/I)) as the second view. All attributes in the dataset contain numerical values. The DBLP dataset contains 6455 authors described by author-conference bipartite graph as the first view and co-authorship network as the second view. All attributes in this dataset have Boolean values. The Phenotype dataset contains 1336 bacterial species. The first view contains attributes containing information about phenotype characteristics of different bacterial species while the second view contains attributes containing information about the presence of different Clusters of Orthologous Groups (COGs) (Tatusov et al. 1997, 2000) in genomes of these bacterial species. All attributes in this dataset have Boolean values.

We used the CLUS-RM redescription mining algorithm, presented in Mihelčić et al. (2015) to create the sets of redescriptions (see Table 2). To create redescriptions, we did not impose any redundancy restrictions except that all redescriptions must be distinct. This is done to demonstrate all capabilities of the tool and enhance construction of different visualizations used for exploration. A more detailed description of algorithm parameters is available in Sect. 2 of Online resource 2.

Full descriptions of all attributes are available in Online resource 1. More detailed redescription set statistics obtained on all three datasets (Table 3) include: the number of redescriptions contained within a set $|\mathcal{R}|$, the average size of a redescription query constructed on a first view $\overline{\left|q_{1}\right|}$, the average size of a redescription query constructed on the second view $\overline{\left|q_{2}\right|}$, the average support size of a redescription $\overline{|\operatorname{supp}(R)|}$, the average accuracy 
Table 3 Number of attributes and simple statistics of redescription sets created on a Country dataset (C), DBLP dataset (D) and Phenotype dataset (P)

\begin{tabular}{lrrlllllll}
\hline Data & $\left|V_{1}\right|$ & $\left|V_{2}\right|$ & $|\mathcal{R}|$ & $\overline{\left|q_{1}\right|}$ & $\overline{\left|q_{2}\right|}$ & $\mid \overline{\operatorname{supp}(R) \mid}$ & $\overline{J(R)}$ & $\overline{A E J(R)}$ & $\overline{A A J(R)}$ \\
\hline$C$ & 49 & 312 & 5488 & 3.89 & 6.75 & 31.29 & 0.69 & 0.11 & 0.07 \\
$D$ & 304 & 6455 & 3674 & 6.37 & 4.86 & 222.88 & 0.29 & 0.05 & 0.09 \\
$P$ & 332 & 4601 & 6200 & 3.13 & 3.75 & 18.05 & 0.7 & 0.08 & 0.03 \\
\hline
\end{tabular}

of redescriptions contained within a set $\overline{J(R)}$, the average entity Jaccard index of redescriptions contained within a set $\overline{A E J(R)}$ and the average attribute Jaccard index of redescriptions contained within a set $\overline{A A J(R)}$.

The SOM displayed in this work was obtained with the R package kohonen (Wehrens and Buydens 2007). To train it, we used 1000 iterations with the learning rate linearly declining from 0.05 to 0.01 . SOM integrated in the tool is a different, JavaScript implementation of the SOM algorithm and can be used independently.

\section{Redescription set exploration with the InterSet tool}

In this section, we describe the redescription set exploration process provided by the InterSet tool (Fig. 1). ${ }^{3}$ It enables targeted and contextual redescription set exploration. Targeted exploration allows effective redescription selection (based on redescription entities, attributes, properties) useful when researchers pursue some hypothesis. Contextual analyses help with interpretation and understanding of different redescriptions by providing different means of placing these redescriptions into a broader context (connect them with similar redescriptions with respect to some criteria), relate them with some other potentially interesting redescriptions and analyse them at the individual level.

The exploration can be done based on any combination of: (i) entities described in redescriptions from the redescription set through the SOM visualization (EC-aspect), (ii) attributes used in redescriptions to describe different entities through the heatmap visualization (AI-aspect) and (iii) quality measures assigned to individual redescriptions by using crossfilter on multiple redescription quality criteria (RQ-aspect).

To construct the SOM visualization, we use the intuition that the high overlap of entities described by redescription queries indicates the existence of shared properties and possible associations between the used attributes. The SOM groups entities based on their membership in support sets of redescriptions contained in a redescription set. Resulting groups potentially share many common properties and are interesting for exploration. In addition, we obtain a spatial map of entities (clusters of entities are arranged in a hexagonal map) based on similarity of their shared properties across both views.

Attribute co-occurrence in redescription queries is used to create a heatmap of crossview pairwise attribute associations (usually observed when more than one view is used). As all redescription mining algorithms focus on discovering redescriptions that describe different subsets of entities, frequent attribute co-occurrence in redescriptions contained in a redescription set could indicate associations (even correlations) of these attributes based on values of some larger subset or even all entities contained in the dataset.

3 All figures depicting functionality of the InterSet where created using several screen shots. Components of the tool were re-arranged in these figures to make them compact and all components visible. 


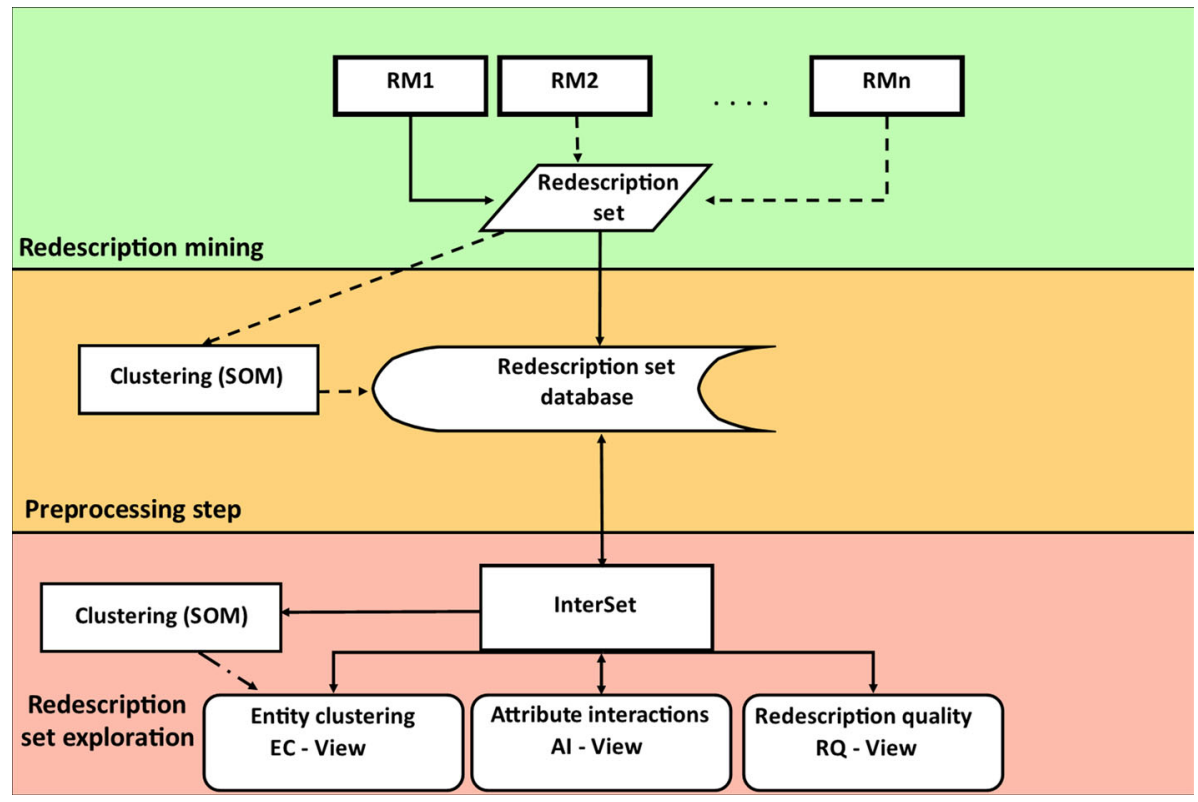

Fig. 1 Schematic description of the process that leads to interactive exploration of redescription sets: (1) Redescription set with one (mandatory) or more (optional) redescription mining algorithms must be generated, (2) preprocessing step involves the use of Self organizing Maps clustering algorithm (optional) and database preparation (mandatory), necessary to perform (3) interactive redescription set exploration with the InterSet tool. Using the clustering with SOM is mandatory for the EC-aspect in the redescription set exploration if it is not done in the pre-processing step

The crossfilter visualization allows selecting smaller subsets of redescriptions using simultaneously several or all defined redescription quality criteria. It is extendible with newly created measures derived from individual redescription properties or some global properties of the redescription set containing these redescriptions (but these measures need to be computed and provided as the input to the tool). In addition, crossfilter displays distributions of the quality criteria of the selected subset of redescriptions in the form of histograms.

Although large number and higher interconnectivity of redescriptions contained in a redescription set might not be a desired property for the final user in a general case, the InterSet can use large diversity and potentially higher interconnectivity in the redescription set to increase the usefulness of exploration. Potential redundancy is used to provide additional information through statistical analyses which enables construction of the SOM and the heatmap visualization (redundant, but non-identical redescriptions reinforce each other). The tool is equipped with the filtering mechanism that allows reducing the level of redundancy, or completely removing redundant redescriptions when the appropriate selection has been made.

Redescriptions contained in the redescription set can also be analysed on the individual level. This includes the value distribution analysis of entities contained in the redescription support, for all attributes contained in redescription queries, and comparison with value distributions of all entities in the dataset or in some more specific groups, such as attribute numeric interval. Violin/notched box plots, used in the tool, allow visualization of irregular distribution shapes, that often arise, when disjunction and negation operators occur in redescription queries. Complex queries, transformed to the Disjunctive normal form (DNF), can be analysed by exploring parts of queries represented as clauses. 


\subsection{Entity-based exploration}

Redescription support sets can not usually be arranged in a strictly hierarchical structure. Rather, they can be highly overlapping with the level of overlap depending on the underlying data, number of redescriptions in the redescription set and the algorithm used to create redescriptions. With such general structure of redescription support sets, we decided to use the Self Organising Map (Kohonen et al. 2001) to enable entity based exploration as it groups entities based on similarities and embeds similar groups closer together on a $2 \mathrm{D}$ visualization map. It allows representing entities from potentially large datasets in a compact form where each entity is a member of only one SOM cluster.

For a given redescription set $\mathcal{R}$ such that $|\mathcal{R}|=n$ and the original dataset containing $m$ entities, we construct a $m \times n$ matrix $A$. The rows of $A$ correspond to the entities from the original dataset, and columns correspond to the redescriptions from the redescription set. Thus, $A_{i j}=1$ if and only if $R_{j} \in \mathcal{R}$ describes an entity $e_{i} \in E$. We use the matrix $A$ to construct a SOM map, containing a user-defined layout of dimensions $w \times h$. As a result, we obtain a partition of the entity set $E=C_{1} \cup C_{2} \cup \cdots \cup C_{k}$, for $k \leq w \cdot h$ and $C_{i} \cap C_{j}=\emptyset, i, j \leq w \cdot h$. If $k<w \cdot h$ then there exist empty clusters which are displayed on the SOM layout but have no functionality.

Our approach to use the SOM for exploration of redescriptions differs from that of CastilloRojas et al. (2014), that uses the SOM to visualize the support of an individual association rule. We utilise the matrix of entity occurrence in support sets of individual redescriptions from the redescription set to construct the SOM. This allows us to obtain a map of entities that share many properties in both views, which is used for targeted and contextual redescription set exploration.

On the presented use case of world countries (see Fig. 2), we have constructed a $4 \times 4$ SOM map to obtain some interesting clusters of entities and their spatial location. For a given cluster $C_{i}$, the set of all redescriptions associated with it is defined as:

$$
r\left(C_{i}\right)=\left\{R_{j} \in \mathcal{R}, \operatorname{supp}\left(R_{j}\right) \cap C_{i} \neq \emptyset\right\}
$$

The cluster marked with a red square box in Fig. $2\left(C_{s e l}\right)$ contains 11 European countries: Austria, Belgium, Denmark, Finland, France, Germany, Italy, Spain, Sweden, Switzerland and United Kingdom. The $\left|r\left(C_{\text {sel }}\right)\right|=2737$, since 2737 redescriptions from $\mathcal{R}$ describe at least one country from $C_{s e l}$. Exploration involves selecting different SOM clusters which shows additional information about this cluster: entities contained in the SOM cluster (6), a subset of redescriptions describing at least one entity (7) and a WordNet (8) containing most frequently occurring words in attribute descriptions.

Detailed explanation of use and functionality of entity-based exploration can be seen in Sect. 3.1 of Online resource 2 .

\subsection{Attribute-based exploration}

Discovering relevant associations between different variables is an essential part of scientific discovery in many research fields such as biology, pharmacy, medicine, physics etc. Associations between different pairs of attributes can be explored with the InterSet by observing co-occurrence frequency of attributes in redescription queries (Fig. 3). In this exploration aspect, we compute the co-occurrence frequency in redescription queries for each pair of attributes $a_{i}, b_{j}, i \leq\left|V_{1}\right|, j \leq\left|V_{2}\right|$. The co-occurrence is computed as: 


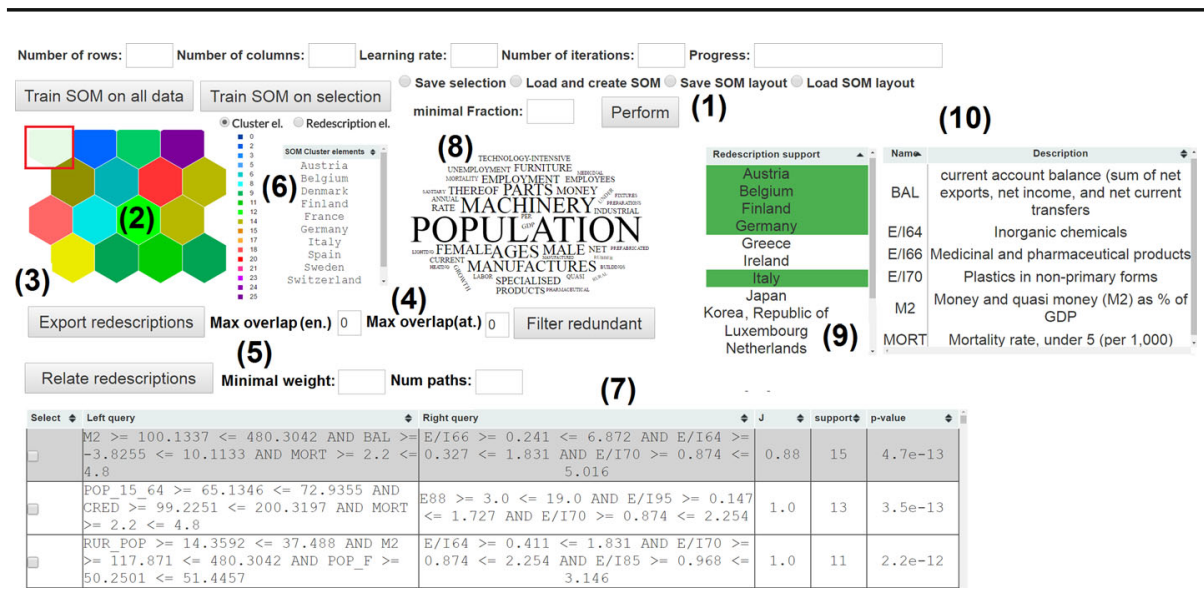

Fig. 2 The entity based interface of the InterSet tool

$$
\operatorname{cooc}\left(a_{i}, b_{j}\right)=\sum_{R_{k}=\left(q_{1_{k}}, q_{2_{k}}\right) \in \mathcal{R}} \mathbb{1}_{a_{i} \in \operatorname{attrs}\left(q_{1_{k}}\right) \wedge b_{j} \in \operatorname{attrs}\left(q_{2_{k}}\right)}
$$

For instance, $\operatorname{cooc}\left(E / I_{83}, P O P_{64}\right)=878$ (see Fig. 3).

In cases where redescription queries contain only conjunction operators in a combination with negations of individual variables, some attribute $a t t_{a}^{b}$, or $a t t^{b}$ is added to the redescription query only if it describes all entities from redescription support and increases the overall redescription accuracy (thus it adds some constraint to the query). Thus, there is a level of association between attributes in such queries on entities contained in the redescription support set. If some pair of attributes occurs often in a redescription set, given the fact that redescription mining algorithms aim to produce redescriptions describing diverse and distinct but overlapping sets of entities, the amount of entities on which some association exists increases significantly, making it potentially interesting for exploration. This approach can capture different kinds of linear or non-linear associations which makes it well suited for different applications. For instance, it is known that there exist highly non-linear associations in the field of biology, which makes them troublesome to detect and analyse. The second advantage is that this representation allows iterative analysis of associations between multiple attributes (which gets prohibitively expensive with standard approaches, such as correlation analysis). An example of detecting associations when dealing with two correlated attributes can be seen in Sect. 8 of Online resource 2 .

If redescriptions contain many disjunction operators, or negations of parts of queries, this connection is not necessarily as strong among all attributes contained in the queries and depends on the redescription creation mechanism of the underlying algorithm. In such cases, the co-occurrence provides optimistic estimate of potentially associated attributes. Generally, redescriptions with queries containing completely disjoint clauses connected with disjunction operators are not considered very useful (because they do not relate some specific information but can instead describe anything) and were are not contained in our redescription sets. Examples of such analyses can be seen in Sect. 7.2 and in Mihelčić and Šmuc (2016). Cooccurrence measures providing better estimates of attribute association can be developed by transforming all redescription queries into Disjunctive Normal Form (DNF) and computing the Jaccard index of support sets of all cross-query pairs of clauses occurring in redescription queries to estimate the level of association. 


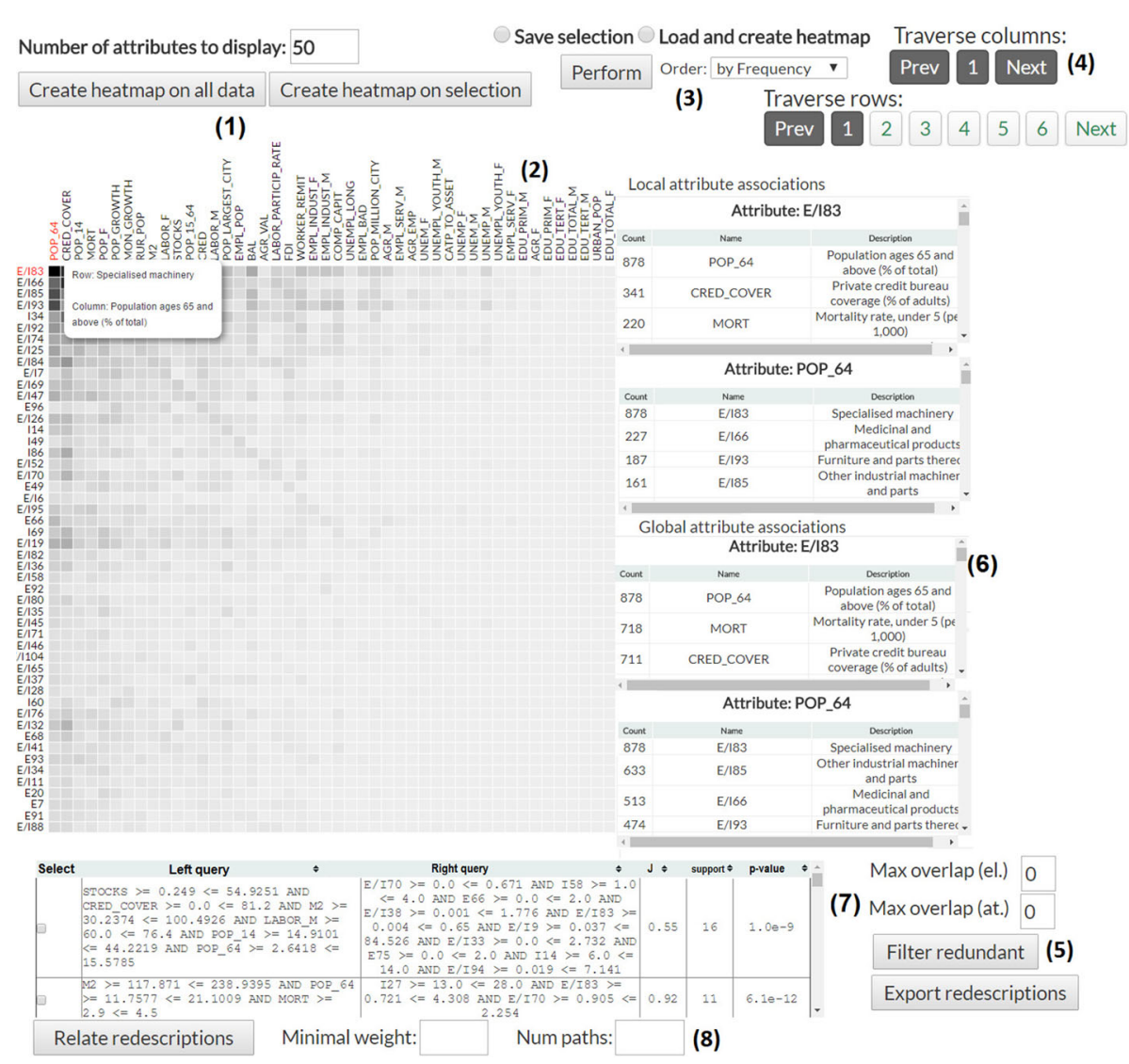

Fig. 3 The attribute based interface of the InterSet tool

The main visualization and exploration component of the attribute based redescription set exploration is the heatmap (Control 2 in Fig. 3). It is displayed as a $k \times k$ matrix where columns represent $k$ attributes from the first view and rows $k$ attributes from the second view. The number $k$ can be defined from Control (1). In our example, we use $50 \times 50$ heatmap. The heatmap can be created by using all redescriptions from $\mathcal{R}$ with create heatmap on all data from Control (1), on redescriptions containing a selected pair of attributes with create heatmap on selection or on arbitrary redescription selection with load and create heatmap option. For a selected pair of attributes $\left(a_{i}, b_{j}\right)$ and a set of redescriptions $\mathcal{R}, \mathcal{R}_{a_{i}, b_{j}} \subseteq \mathcal{R}$ denotes a set of redescriptions:

$$
\mathcal{R}_{a_{i}, b_{j}}=\left\{R=\left(q_{1}, q_{2}\right) \in \mathcal{R} \mid a_{i} \in \operatorname{attrs}\left(q_{1}\right) \wedge b_{j} \in \operatorname{attrs}\left(q_{2}\right)\right\}
$$

Redescription set $\mathcal{R}_{E / I_{83}, P O P_{64}}$ contains 878 redescriptions. By iteratively selecting pairs of attributes and creating a heatmap by using only selected redescriptions, users can simultaneously analyse potential associations among larger sets of attributes and steer the search towards interesting subsets of redescriptions. Additional information about the attributebased exploration aspect can be found in Sect. 3.2 of Online resource 2 .

The SOM based representation can also be applied to attributes used in redescription queries similarly as it was applied to entities. The main advantage of using the SOM is that 


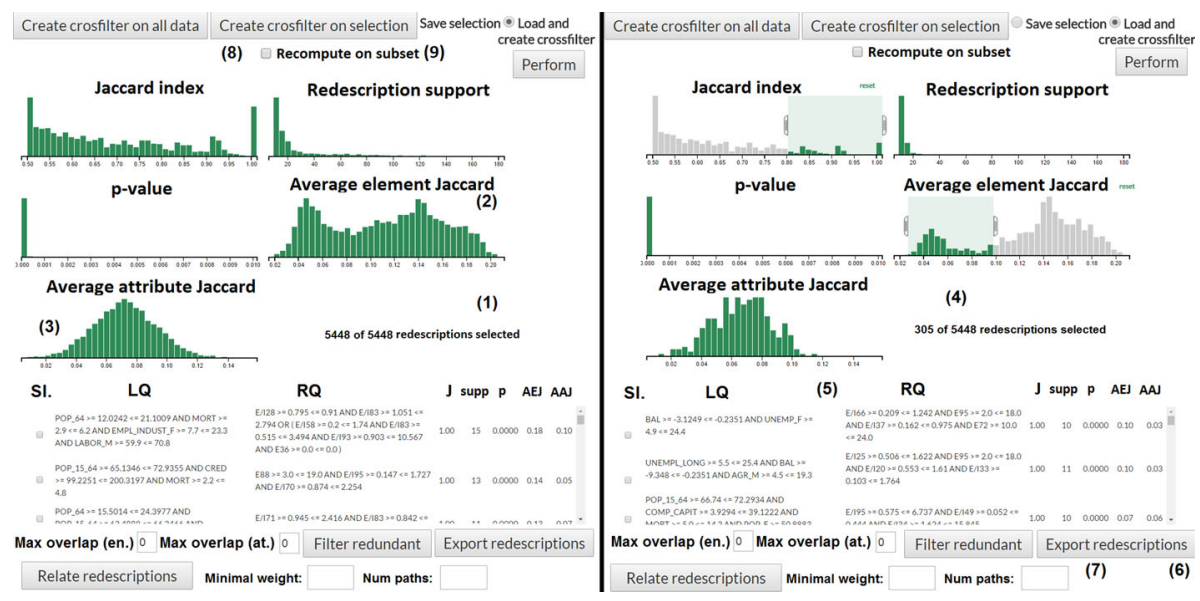

Fig. 4 The InterSet interface based on redescription properties. Initial configuration is shown in the left and the filtering step in the right part of the figure

it instantaneously reveals interactions of more than two different variables. However, it is not possible to distinguish between views in such visualization and it is hard to explore associations between the neighbouring groups of attributes. It is also hard to achieve very focused attribute exploration that can be achieved using heatmap. The heatmap visualisation enables exploring interactions between all cross-view attribute pairs and ordering rows and columns based on different criteria, which is the main reason for our choice. The focus is on cross-view relations since these are usually interesting when exploring similarities based on different contexts, though it can also be used to show all co-occurrence frequencies.

\subsection{Property-based exploration}

The exploration aspect presented in this Section is based on different redescription properties (see Fig. 4). These properties can be computed from the individual redescriptions or derived by using information about the whole redescription set.

Property-based exploration enables fine-grained filtering of a given set of redescriptions by using one or more user-defined criteria. The goal is to reduce the size of a redescription set and obtain a new set that is easier to explore and contains only redescriptions with selected properties. The exploration aspect uses sliders as in the work from Blanchard et al. (2003), but also contains a crossfilter (Control 1 in Fig. 4), which allows instantaneous display of value distribution of different criteria for redescriptions contained in the filtered redescription set (Control 4). The corresponding redescriptions can be explored through Table 5. Crossfilter instantaneously updates Table 5. as user slides through the values of one or more different criteria (Control 4) and displays distribution of all other criteria in a form of histograms. Distributions displayed in the crossfilter provide some high level information about the underlying data (what is the size of clusters obtained, how many highly accurate redescriptions are present in the set etc.). The visualization can be efficiently used with redescription sets containing larger number (several thousand) of redescriptions which are much harder to represent with some other visualization techniques such as parallel coordinate plots. In this work, we use several redescription quality criteria currently found in the literature (Galbrun 2013; Mihelčić et al. 2015). 
The average entity Jaccard index (Control 2) and the average attribute Jaccard index (Control 3) presented in Sect. 2 can be used to extract redescriptions describing (in)frequently described sets of entities or that containing (in)frequent combination of attributes depending on the crossfilter setting. The selection provides no guarantees on entity or attribute overlap between pairs of redescriptions in the newly constructed set. However, filtering (described in Sect. 3.1 of Online resource 2) reduces this overlap to user defined level (Control 7).

For a given set of redescriptions $\mathcal{R}$ and a selected set of filtering constraints $\mathcal{F}=\{J \in$ $\left[j_{\text {min }}, j_{\text {max }}\right]$, supp $\in\left[s_{\text {min }}, s_{\text {max }}\right], p \in\left[p_{\text {min }}, p_{\text {max }}\right], A E J \in\left[a e j_{\text {min }}, a e j_{\text {max }}\right], A A J \in$ $\left.\left[a a j_{\min }, a a j_{\max }\right]\right\}$, we define a set of redescriptions:

$$
\begin{array}{r}
\mathcal{R}_{\mathcal{F}}=\left\{R \in \mathcal{R} \mid j_{\text {min }} \leq J(R) \leq j_{\text {max }}, s_{\text {min }} \leq \operatorname{supp}(R) \leq s_{\text {max }}, p_{\text {min }} \leq p(R) \leq\right. \\
\left.p_{\text {max }}, a e j_{\text {min }} \leq A E J(R) \leq a e j_{\text {max }}, a a j_{\text {min }} \leq A A J(R) \leq a a j_{\text {max }}\right\}
\end{array}
$$

In the provided example (see Fig. 4), the filtering constraints used are $j_{\min }>0.8$ and $a e j_{\text {min }} \leq 0.1$. The resulting set contains 305 redescriptions.

A detailed description of all controls in property-based exploration aspect is available in Sect. 3.3 of Online resource 2 .

\subsection{Interaction between exploration aspects}

It is often useful to combine different exploration techniques to obtain the desired subset of redescriptions. InterSet allows using any combination of previously defined aspects by enabling users to save the selected subset of redescriptions, obtained by using any exploration aspect to the database (with the save selection option, see Figs. 2, 3 and 4). The saved subset of redescriptions can be loaded into arbitrary exploration aspect and used to create the corresponding visualization (SOM, heatmap, crossfilter). This allows viewing interesting attribute associations that occur in redescriptions associated with some SOM cluster, or creating SOM map by using only highly accurate redescriptions.

Interaction between aspects through a database allows using exploration aspects independently until a subset of interest is located and it alleviates various computational problems that would occur if such interaction would be done directly (e.g it is not feasible to recompute a SOM on every filter move).

One example of using such interaction is presented in Sect. 4 of Online resource 2.

\subsection{Relating a sequence of redescriptions}

In this section, we describe the process of relating a sequence of redescriptions $R_{\text {start }}, R i_{1}$, $R i_{2}, \ldots, R_{\text {end }}$, where each redescription occurs only once. Users can choose this sequence by checking the checkbox in the row containing the desired redescription (see Figs. 2, 3 and 4). The selection order is important, since the first selected redescription is marked as the starting node, the last selected redescription as the end node and all other selected redescriptions as intermediary nodes (in order of selection). To be able to relate a sequence of redescriptions based on sets of described entities, we create the adjacency matrix $A$ of dimensions $|\mathcal{R}| \times|\mathcal{R}|$. The element at the index $a_{i, j} \in A$ denotes $J\left(\operatorname{supp}\left(R_{i}\right), \operatorname{supp}\left(R_{j}\right)\right)$. The adjacency matrix is computed only once and then stored in the database. This graph representation is used to find the top- $k$ shortest paths starting from the first selected redescription, ending at the last selected redescription and connecting all other redescriptions in selection order. These paths may contain additional redescriptions having positive entity Jaccard index with redescriptions contained in the initially selected sequence. 
The algorithm for relating a sequence of redescriptions is described in Algorithm 1. To obtain the shortest paths containing a sequence of redescriptions with a given starting and destination node, we use the Dijkstra's shortest path algorithm (Dijkstra 1959) (function findShortest Path, lines 3 and 13 in Algorithm 1). For a selected sequence of redescriptions $R_{\text {start }}, R i_{1}, R i_{2}, \ldots, R_{\text {end }}$, we apply the Dijkstra's algorithm sequentially on each pair of redescriptions $\left(\left(R_{\text {start }}, R i_{1}\right),\left(R i_{1}, R i_{2}\right), \ldots,\left(R i_{k}, R_{\text {end }}\right)\right)$ and concatenate into one large path (lines 3, 13 in Algorithm 1). The forbEdge parameter, forbids the algorithm to use a given set of edges in path construction. We further modified the Dijkstra's algorithm by adding the minimal overlap constraint between pairs of redescriptions (as in story telling). This constraint disables edges with inadequate overlap to be present on the shortest paths in the relation graph. This, modified version of Dijkstra's algorithm is incorporated into the variant of Yen's algorithm $(1971,1972)$ (lines 2-24 in Algorithm 1) to construct the $k$-shortest paths starting at $R_{\text {start }}$, ending at $R_{\text {end }}$ and connecting all redescriptions from the initially selected sequence of redescriptions.

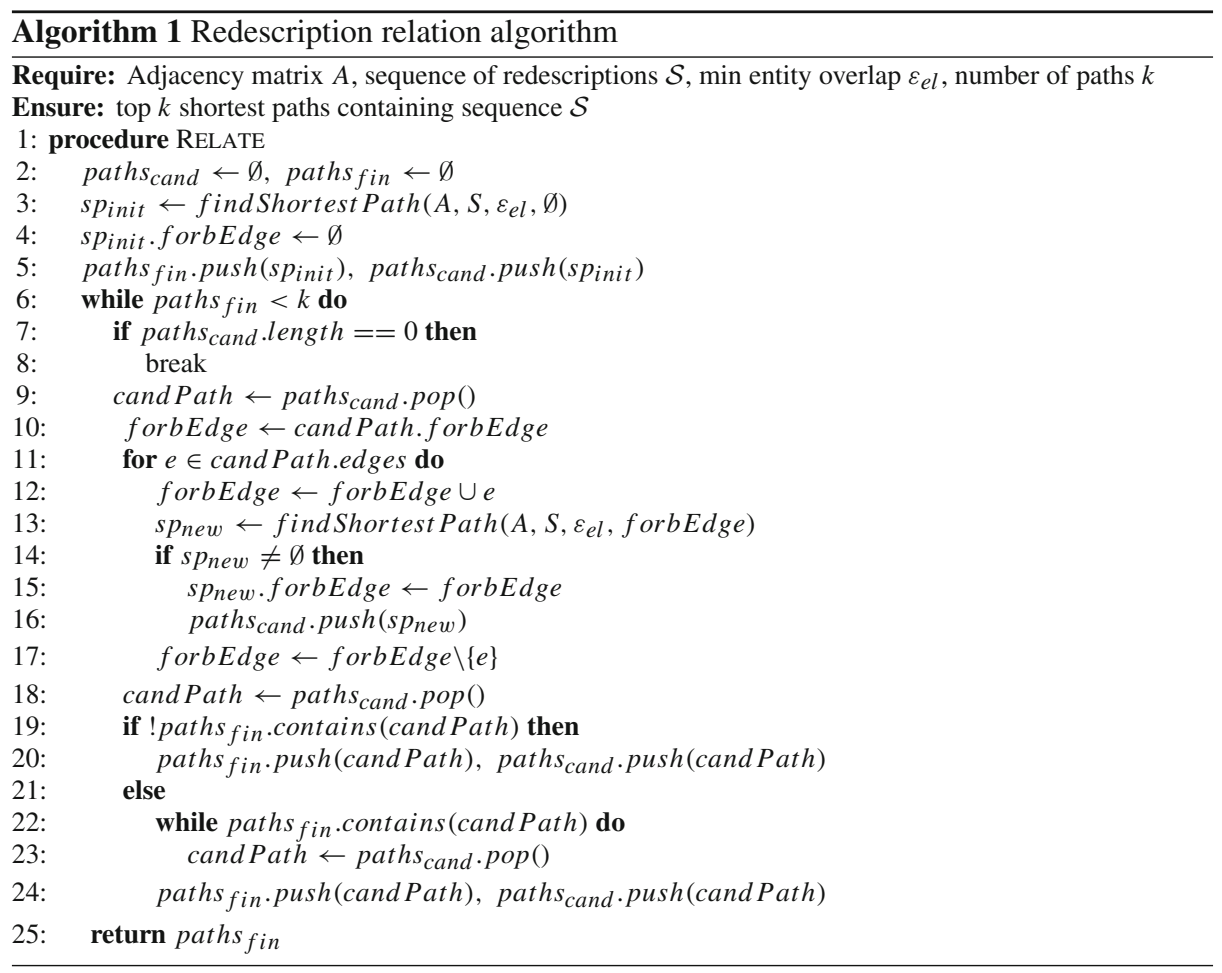

The algorithm finds the shortest path containing the predefined sequence of redescriptions (line 3 in Algorithm 1), then disables one edge at a time on this path (lines 11, 12 in Algorithm 1) to find the second shortest path etc. The procedure is repeated until the top $k$ shortest paths have been found or no new paths can be generated. Variables paths $s_{\text {cand }}$ and paths fin are priority queues which sort the generated paths by distance, placing shorter paths in the front of the queue. 
Relating very similar redescriptions with respect to described entities ideally enables discovery of a) new properties of a set of entities by observing diferent redescriptions describing very similar subset of entities, b) finding redescriptions sharing properties of two or more different redescriptions, c) locating a subset of entities satisfying properties of multiple redescriptions. We denote such set of redescriptions a closer or broader neighbourhood of a redescription. Relating apparently unrelated redescriptions is also interesting and studied in more detail in a data mining task called story telling (Kumar et al. 2008). Depending on a quality of available redescription set, our procedure can find more relaxed sequences of redescriptions (potentially containing mutually different queries) or stories if present in a redescription set. Understanding relations between apparently disjoint redescriptions is interesting in many scientific fields, most notably biology (e.g to relate a set of genes expressed in one experiment to another set, implicated in a different pathway, to observe potential connections between two apparently unrelated phenotypes, etc.).

The discovered top $k$ shortest paths containing a predefined sequence of redescriptions are visualized in a form of a graph. The nodes of a relation graph represent redescriptions and contain information about the redescription properties and queries (displayed on mouse hover). Edges contain information about the level of overlap between two redescriptions, colour denotes the edge membership to the shortest path and the number denotes the rank of the path containing the selected edge. If multiple paths share an edge, it is coloured by the colour of the best ranking path.

The advantage of our visualization and exploration technique is that it allows relating more than a pair of redescriptions and embeds (potentially multiple) paths in a relation graph which provides compact and interpretable visual information to the user. It is important to note that for each generated path and for entities described by neighbouring redescriptions in the sequence, the following relations apply: $R_{\text {start }} \Leftrightarrow R_{i_{1}} \Leftrightarrow R_{\text {new } 1} \Leftrightarrow R_{i_{2}} \Leftrightarrow \cdots \Leftrightarrow R_{\text {end }}$. Thus, we are able to provide redescriptions whose queries are actually redescriptions (a construct previously unavailable in data mining). For instance $R_{r}=\left(R_{\text {start }}, R_{i_{i}}\right)$ is a redescription containing redescriptions as its queries. Such complex constructs provide higher order information about properties of some subset of entities (not easily observable by analyses of individual redescriptions).

We demonstrate the function of this visualization by relating a sequence containing three redescriptions $R_{s}, R_{i_{1}}, R_{e}$ (see Table 4). The example uses redescriptions obtained on the Country dataset (described in Sect. 5).

For these redescriptions, we create the relation graph containing $k$-paths, $1 \leq k \leq 4$ (demonstrated in Fig. 5). All produced edges are required to have a weight larger or equal to 0.4 .

As we can see from Fig. 5, redescriptions $R_{S}$ and $R_{i_{1}}$ are quite different from redescription $R_{e}$ (absence of edges between them indicates their overlap is less than 0.4 ). The procedure however, managed to find one additional redescription (denoted $R_{\text {new } 1}$ ) with overlap 0.71 with redescription $R_{e}$ and an overlap of 0.5 with redescription $R_{i_{1}}$. The shortest path discovered is $R_{s}, R_{i_{1}}, R_{\text {new } 1}, R_{e}$. Other generated paths, instead of going directly from $R_{S}$ to $R_{i_{1}}$, find additional redescription with very high similarity with redescriptions $R_{S}$ and $R_{i_{1}}$ and use it as an additional node in the path. The second discovered shortest path equals $R_{s}, R_{\text {new } 2}, R_{i_{1}}, R_{\text {new } 1}, R_{e}$. Newly added redescriptions are chosen to have as high as possible similarity to the constraint points (starting, intermediary, end nodes) on the path. Redescription $R_{\text {new } 2}$ has high entity similarity with $R_{s}$ and $R_{i_{1}}$ while $R_{\text {new } 1}$ has high entity similarity with $R_{i_{1}}$ and $R_{e}$.

For demonstration, we analyse the case $k=1$ and redescription $R_{n e w_{1}}$ defined in Table 5 . 
Table 4 Redescriptions $R_{S}, R_{i_{1}}$ and $R_{e}$

\begin{tabular}{|c|c|}
\hline$R_{S}:$ & $\left(q_{1_{s}}, q_{2_{s}}\right)$ \\
\hline$q_{1_{s}}:$ & $65.2 \leq \mathrm{POP}_{[15,64]} \leq 72.9 \wedge 99.2 \leq \mathrm{CRED} \leq 200.3 \wedge 2.2 \leq \mathrm{MORT} \leq 4.8$ \\
\hline$q 2_{s}:$ & $3.0 \leq E_{88} \leq 19.0 \wedge 0.15 \leq \mathrm{E} / \mathrm{I}_{95} \leq 1.7 \wedge 0.87 \leq \mathrm{E} / \mathrm{I}_{70} \leq 2.25$ \\
\hline \multirow[t]{2}{*}{$\operatorname{supp}\left(R_{S}\right):$} & Austria, Finland, Germany, Greece, Ireland, Italy, Republic of Korea, \\
\hline & Luxembourg, Netherlands, Portugal, Spain, Switzerland, United Kingdom \\
\hline quality: & $J\left(R_{S}\right)=1.0,\left|\operatorname{supp}\left(R_{S}\right)\right|=13, p\left(R_{S}\right)=3.5 \cdot 10^{-13}$ \\
\hline$R_{i_{1}}:$ & $\left(q_{i_{1}}, q 2_{i_{1}}\right)$ \\
\hline$q_{1_{i_{1}}}:$ & $\begin{array}{l}14.4 \leq \mathrm{RUR} . \mathrm{POP} \leq 37.5 \wedge 117.9 \leq \mathrm{M}_{2} \leq 480.3 \wedge 50.3 \leq \mathrm{POP} . \mathrm{F} \\
\quad \leq 51.4\end{array}$ \\
\hline$q 2_{i_{1}}:$ & $0.4 \leq \mathrm{E} / \mathrm{I}_{64} \leq 1.8 \wedge 0.87 \leq \mathrm{E} / \mathrm{I}_{70} \leq 2.3 \wedge 0.97 \leq \mathrm{E} / \mathrm{I}_{85} \leq 3.1$ \\
\hline \multirow[t]{2}{*}{$\operatorname{supp}\left(R_{i_{1}}\right):$} & Austria, Finland, Germany, Ireland, Italy, Switzerland, Republic of Korea, \\
\hline & Luxembourg, Netherlands, Spain, United Kingdom \\
\hline quality: & $J\left(R_{i_{1}}\right)=1.0,\left|\operatorname{supp}\left(R_{i_{1}}\right)\right|=11, p\left(R_{i_{1}}\right)=2.2 \cdot 10^{-12}$ \\
\hline$R_{e}:$ & $\left(q_{1_{e}}, q_{2_{e}}\right)$ \\
\hline$q_{1_{e}}:$ & $\begin{array}{l}15.5 \leq \mathrm{POP}_{64} \leq 24.4 \wedge 62.5 \leq \mathrm{POP}_{[15,64]} \leq 66.3 \wedge 2.8 \leq \mathrm{UNEM} . \quad \mathrm{F} \\
\quad \leq 11.9\end{array}$ \\
\hline$q_{2}:$ & $0.95 \leq \mathrm{E} / \mathrm{I}_{71} \leq 2.4 \wedge 0.84 \leq \mathrm{E} / \mathrm{I}_{83} \leq 7.9 \wedge 13.0 \leq \mathrm{I}_{2} \leq 19.0$ \\
\hline \multirow[t]{2}{*}{$\operatorname{supp}\left(R_{e}\right):$} & Belgium, Denmark, Finland, France, Germany, Italy, Japan, Netherlands, \\
\hline & Norway, Sweden, UK \\
\hline quality: & $J\left(R_{e}\right)=1.0,\left|\operatorname{supp}\left(R_{e}\right)\right|=11, p\left(R_{e}\right)=2.2 \cdot 10^{-12}$ \\
\hline
\end{tabular}

The most important attributes used in the aforementioned redescription examples are explained in Table 6. $E$ denotes a percentage of export comprised by the export of a given commodity, $I$ denotes the percentage of import comprised by the import of a given commodity, $E / I$ denotes the ratio of two aforementioned percentages.

In this example, we locate three redescriptions of interest, $R_{s}, R_{i_{1}}$ and $R_{e}$. Our goal is to find redescriptions sharing the properties of these redescriptions (having similar supports or using similar attributes) or finding redescriptions that reveal new properties about these specific (or very similar) subsets of countries. Obtaining multiple similar redescriptions of some set of entities is possible with a SOM-map, but only if the given set of entities is fully contained within a SOM cluster. Finding multiple redescriptions of subsets of entities shared across multiple SOM clusters is not possible with this approach. Relation graphs allow obtaining such information (as shown in Fig. 5).

The newly discovered (presented) redescription $R_{\text {new }}$ contains similarities with redescriptions $R_{i_{1}}$ and with $R_{e}$. The set $\operatorname{supp}\left(R_{n e w 1}\right)$ contains a mix of countries described by $R_{i_{1}}$ and $R_{e}$. The similarities can be seen also from the queries of $R_{n e w 1}$. Percentage of elderly population (aged 65 and above) is used with similar numerical bounds as in redescription $R_{e}$ and the query containing attributes related to trading patterns in $R_{n e w 1}$ contains disjunction operator, which allows describing different subsets of countries with different parts of queries. The first part of this query contains the same attribute $\left(E / I_{85}\right.$ - export to import ratio of other industrial machinery and parts) with similar numerical interval as in the corresponding query of $R_{i_{1}}$ while the second part of the query contains the same attribute $\left(E / I_{71}\right.$ - export to import ratio of chemical material and products) with similar numerical interval as in the corresponding query of $R_{e}$. In the presented use case, we found 4 additional redescriptions 

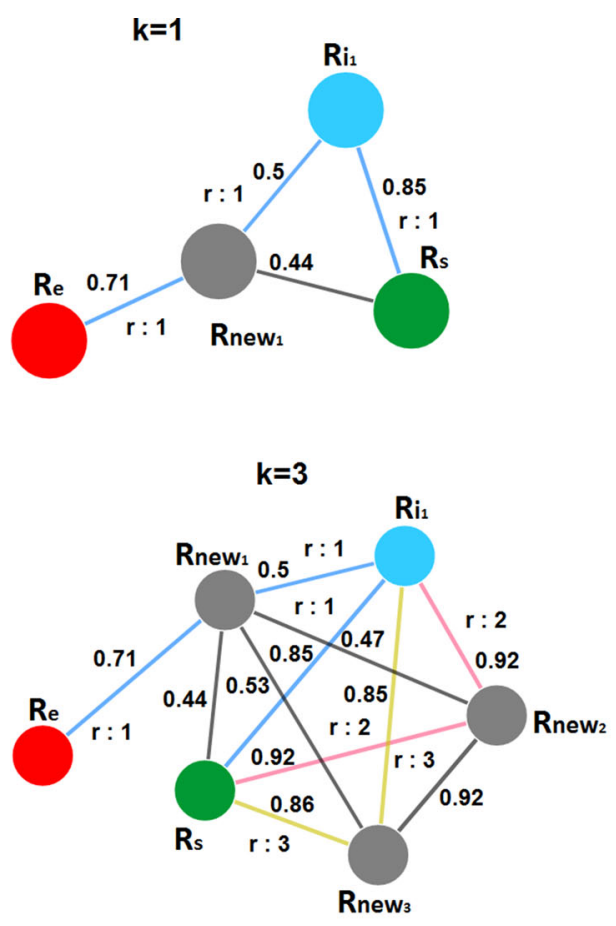

$\mathbf{k}=\mathbf{2}$

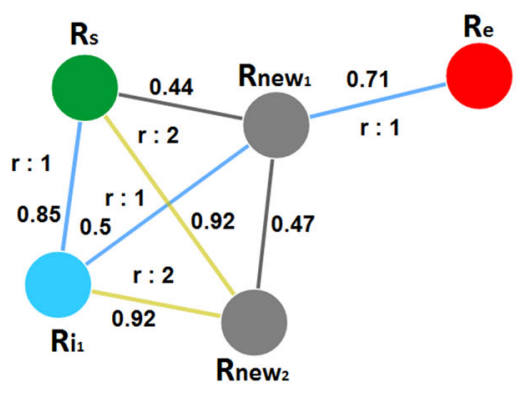

$k=4$

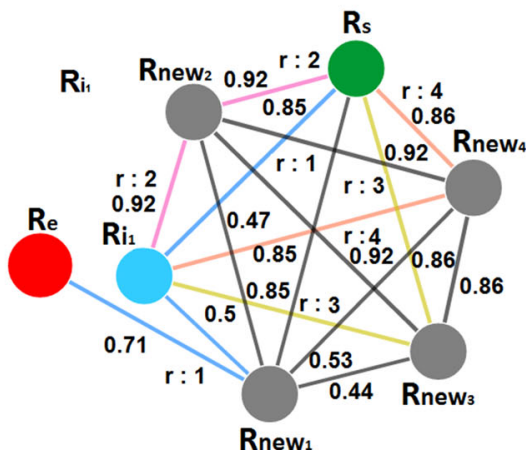

Fig. 5 Relation graphs obtained for the sequence $R_{S}, R_{i_{1}}, R_{e}$ for $1 \leq k \leq 4$ and $\varepsilon_{e l}=\omega_{\min } \geq 0.4$

Table 5 Redescription $R_{\text {new }}$

\begin{tabular}{ll}
\hline$R_{\text {new } 1}:$ & $\left(q_{1_{\text {new } 1}}, q_{2_{\text {new } 1}}\right)$ \\
$q_{1_{\text {new } 1}}:$ & $15.5 \leq \mathrm{POP}_{64} \leq 21.1 \wedge-0.4 \leq \mathrm{FDI} \leq 5.4 \wedge 0.1 \leq \mathrm{POP}$. GROWTH $\leq 1.3$ \\
$q_{2_{\text {new } 1}:}$ & $0.56 \leq \mathrm{E} / \mathrm{I}_{2} \leq 1.5 \wedge 0.2 \leq \mathrm{E} / \mathrm{I}_{93} \leq 0.7 \wedge 0.76 \leq \mathrm{E} / \mathrm{I}_{85} \leq 1.57 \vee$ \\
& $\left(0.9 \leq \mathrm{E} / \mathrm{I}_{93} \leq 3.5 \wedge 0.95 \leq \mathrm{E} / \mathrm{I}_{71} \leq 1.9\right)$ \\
$\operatorname{supp}\left(R_{\text {new } 1}\right):$ & Austria, Belgium, Denmark, Finland, France, Germany, Italy \\
& Netherlands, Norway, Spain, Sweden, Switzerland, United Kingdom \\
quality: & $J\left(R_{e}\right)=0.93,\left|\operatorname{supp}\left(R_{e}\right)\right|=13, p\left(R_{e}\right)=9.3 \cdot 10^{-13}$ \\
\hline
\end{tabular}

with very high entity similarity to both $R_{S}$ and $R_{i_{1}}$. In the economic use-case of analysing the trading success and wealth of a country, we could select one or more redescriptions of highly developed countries and relate them with one or more redescriptions of underdeveloped or countries under development. Observing newly presented redescriptions could potentially reveal specific reasons (trade or socio-demographic) that distinguish one set of countries from another, but could also offer a potential guideline in how to improve the status of underdeveloped countries and countries under development.

All attributes used in redescription queries are explained in Online resource 1. 
Table 6 Descriptions of attributes occurring in redescriptions $R_{\text {start }}, R_{i_{1}}$ and $R_{\text {end }}$

\begin{tabular}{|c|c|}
\hline Attribute & Description \\
\hline $\mathrm{POP}_{64}$ & Percentage of total population aged 65 and above \\
\hline $\mathrm{POP}_{[15,64]}$ & Percentage of total population aged 15 to 64 \\
\hline POP. GROWTH & Percentage of annual population growth \\
\hline CRED & Domestic credit to private sector \\
\hline MORT & Mortality rate under 5 (per 1000) \\
\hline RUR. POP & Percentage of total population living in rural area \\
\hline $\mathrm{M}_{2}$ & Percentage of GDP as money and quasi money \\
\hline POP. F. & Percentage of total population comprised by females \\
\hline UNEM. F. & Percentage of unemployed female work force \\
\hline FDI & Foreign direct inflows \\
\hline $\mathrm{E}_{88}$ & $E$ of electrical machinery, apparatus and appliances \\
\hline $\mathrm{I}_{2}$ & $I$ of primary commodities, excluding fuels \\
\hline $\mathrm{E} / \mathrm{I}_{2}$ & $E / I$ of primary commodities, excluding fuels \\
\hline $\mathrm{E} / \mathrm{I}_{64}$ & $E / I$ of essential oils for perfume materials and cleaning preparations \\
\hline $\mathrm{E} / \mathrm{I}_{70}$ & $E / I$ of Leather, leather manufactures and dressed fur skins \\
\hline $\mathrm{E} / \mathrm{I}_{71}$ & $E / I$ of Rubber manufactures \\
\hline $\mathrm{E} / \mathrm{I}_{83}$ & $E / I$ of office machines and automatic data processing machines \\
\hline$E / I_{85}$ & $E / I$ of electrical machinery, apparatus and appliances \\
\hline $\mathrm{E} / \mathrm{I}_{93}$ & $E / I$ of primary commodities, precious stones and non-monetary gold \\
\hline $\mathrm{E} / \mathrm{I}_{95}$ & $E / I$ of total all products \\
\hline
\end{tabular}

\subsection{Individual redescription analysis}

The most specific type of analysis provided by the InterSet is the analysis of individual redescriptions. The goal of this analysis is to deepen the overall understanding of the redescription queries and consequently their associations. To enable detailed analysis, the tool requires redescription queries to be transformed in the Disjunctive normal form (DNF). This decomposed form allows analysing distribution of a subset of redescription support described by each clause in this representation. Since the general goal is to produce short, understandable queries and every formula in Propositional logic can be transformed to an equivalent DNF (Kroening and Strichman 2008), it is a reasonable and mostly feasible requirement aimed at increasing understandability. Depending on the type of attributes, distributions are visualized with notched box plot or violin plots in case of numerical attributes or pie charts for categorical and Boolean attributes. The main idea is to allow users to visually inspect the distributions of different subsets of entities, for a given attribute, and compare them with the distribution of all entities in the dataset. Such procedure may reveal interesting properties (such as unusually high or low values of the corresponding attribute) inciting new research directions. Comparing distributions of entities contained in different clauses reveals the relation between them and provides information about the number of entities described by each clause. Visual comparison must not be taken as evidence of some property and appropriate statistical tests need to be performed to confirm or reject a hypothesis.

If redescription attributes contain numerical values, depending on the query complexity, the analysis contains i) three comparative notched/violin plots if the DNF representation 


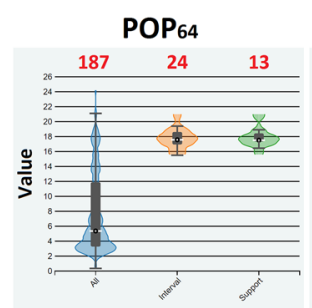

$\mathrm{E} / \mathrm{I}_{3} \mathrm{Cl}_{1}$

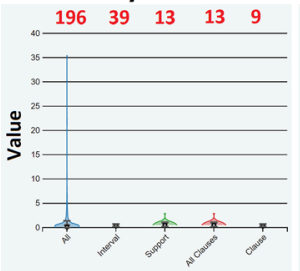

FDI $\mathrm{Cl}_{1}$

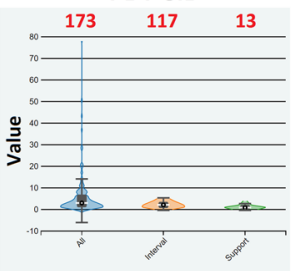

$\mathrm{E} / \mathrm{I}_{85} \mathrm{Cl}_{1}$

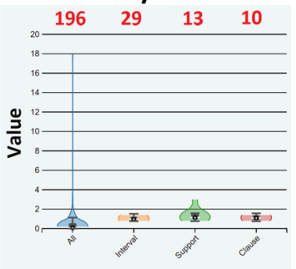

POP. GROWTH Cl

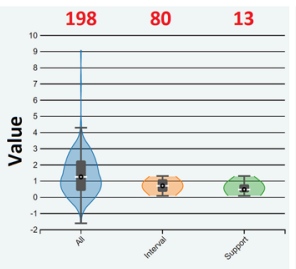

$\mathrm{E} / \mathrm{l}_{93} \mathrm{Cl}_{2}$

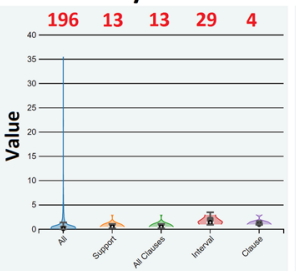

$\mathrm{E} / \mathrm{I}_{2} \mathrm{Cl}_{1}$

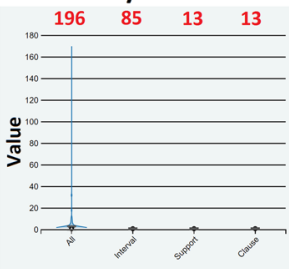

$\mathrm{E} / \mathrm{I}_{71} \mathrm{Cl}_{2}$

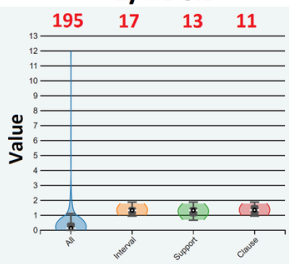

Fig. 6 Comparative violin plots showing entity value distribution for all attributes occurring in the selected redescription $\left(R_{n e w 1}\right)$. The violin plots, from left to right, show entity value distribution: (1) entity value distribution, for the selected attribute, of all entities containing non-missing values in the dataset (blue), (2) entity value distribution, for the selected attribute, of all entities containing non-missing values in a numeric interval defined in a redescription query (orange), (3) entity value distribution, for the selected attribute, of all entities contained in the redescription support set (green), (4) entity value distribution of all entities contained in redescription support set that are described by at least one clause containing the attribute under investigation (red), (5) entity value distribution of all entities contained in the redescription support set described by a particular clause which contains the analysed variable (purple) (Color figure online)

doesn't contain disjunction operators (explanation 1, 2, 3 in Fig. 6), ii) four plots if the DNF representation contains disjunction operators (explanation 1, 2, 3, 5 in Fig. 6) and iii) five plots if an attribute occurs in more than one clause in the DNF representation of a query (explanation 1, 2, 3, 4, 5 in Fig. 6). On hover over the plot, the user gets information about minimal, maximal values, median, and values of a first and the third quartile. Whereas analysis with violin plot provides information about entity distribution and density, using notched box plot allows observing outliers and the confidence interval of the median, which is very important to assess the significance of the provided information about the median value. Regardless of the type of plot used, it is possible to display trend lines which allow visually detecting the differences between medians and quartiles of different plots (further assisting in relating different subsets of entities and comparing their distributions).

Visualization displayed in Fig. 6 depicts entity value distributions, visualized with violin plots, based on queries, query support sets and support set of a redescription $R_{\text {new } 1}$, defined in Sect. 6.5. Visualization using notched box plot with trend lines can be seen in Sect. 5 of Online resource 2.

In case of categorical and Boolean variables, the analysis contains two pie charts. One chart visualizes value distribution of all entities in the dataset, while the second pie chart visualizes distribution of values for entities contained in the redescription support set and compares it to the distribution of entities not contained in the support set for all categories represented by entities from the support set. This provides information about the quantity of entities, with a given categorical value, that are described by the selected redescription. Redescriptions describing entities containing only one or an exclusive subset of categorical values or describing majority of entities containing some categorical value may provide important information about the explored data. We demonstrate the described analysis on a 
Table 7 Redescription $R_{\text {ex }}$

\begin{tabular}{ll}
\hline$R_{e x}:$ & $\left(q_{1_{e x}}, q 2_{e x}\right)$ \\
$q_{1_{e x}}:$ & SymponCompGeometry $\wedge$ CCCG \\
$q_{2_{e x}}:$ & $\neg(\neg$ Ferran Hurtado $\wedge \neg$ Boris Aronov $\wedge \neg$ Godfried T. Toussaint $)$ \\
quality: & $J\left(R_{e x}\right)=0.54, p\left(R_{e x}\right)=0.0,\left|\operatorname{supp}\left(R_{e x}\right)\right|=80$ \\
\hline
\end{tabular}

SymposiumonComputationalGeometry

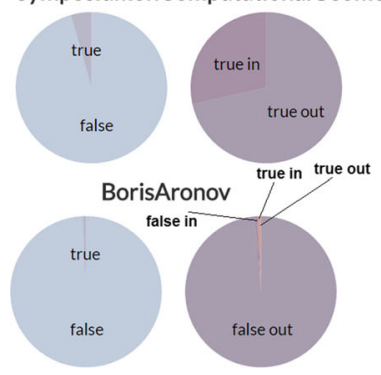

CCCG
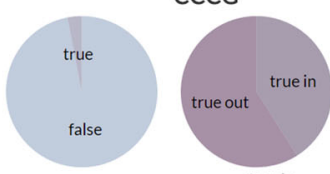

GodfriedT.Toussaint true in true out $^{\text {to }}$ false in

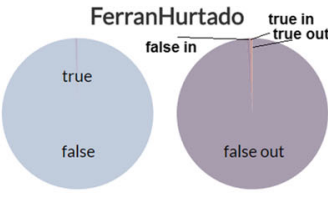

true

false

Fig. 7 Comparative pie charts showing entity value distribution for all attributes occurring in the selected redescription. The pie charts show entity value distribution in order: (1) entity value distribution for all entities containing non-missing value in the dataset, (2) entity value distribution for all entities contained in redescription support compared to the distribution of values for all entities not contained in the support set whose categorical values occur among described entities

redescription $R_{e x}=\left(q_{1_{e x}}, q_{2_{e x}}\right)$ (see Table 7) obtained on the DBLP dataset (introduced in Sect. 5).

Query $q_{2_{e x}}$ is logically equivalent to the query $q_{2_{e x}}^{\prime}=$ Ferran Hurtado $\vee$ Boris Aronov $\vee$ Godfried $T$. Toussaint. Because of this, the $\operatorname{supp}\left(R_{e x}\right)$ may contain entities with values Ferran Hurtado $=$ TRUE and Ferran Hur - tado = FALSE (the same applies for the other authors), thus the second pie chart needs to contain distributions for both categorical values. The distribution analysis is demonstrated in Fig. 7.

\section{Applying InterSet to explore redescription sets}

In this section we demonstrate the use of InterSet tool on three different use case data sets. All visualizations presented (including redescription relation graph) are integrated into the tool, however various statistical tests were computed using external tools and are currently not a part of InterSet.

\subsection{Exploring redescriptions obtained on the Country dataset}

In our previous work (Mihelčić and Šmuc 2016), we discovered many useful and interesting redescriptions using individual exploration aspects in isolation on the Country dataset.

In this work, we demonstrate the exploration by using all aspects in the interactive setting. The use of a redescription relation graph on the Country dataset is already demonstrated in Sect. 6.5. 


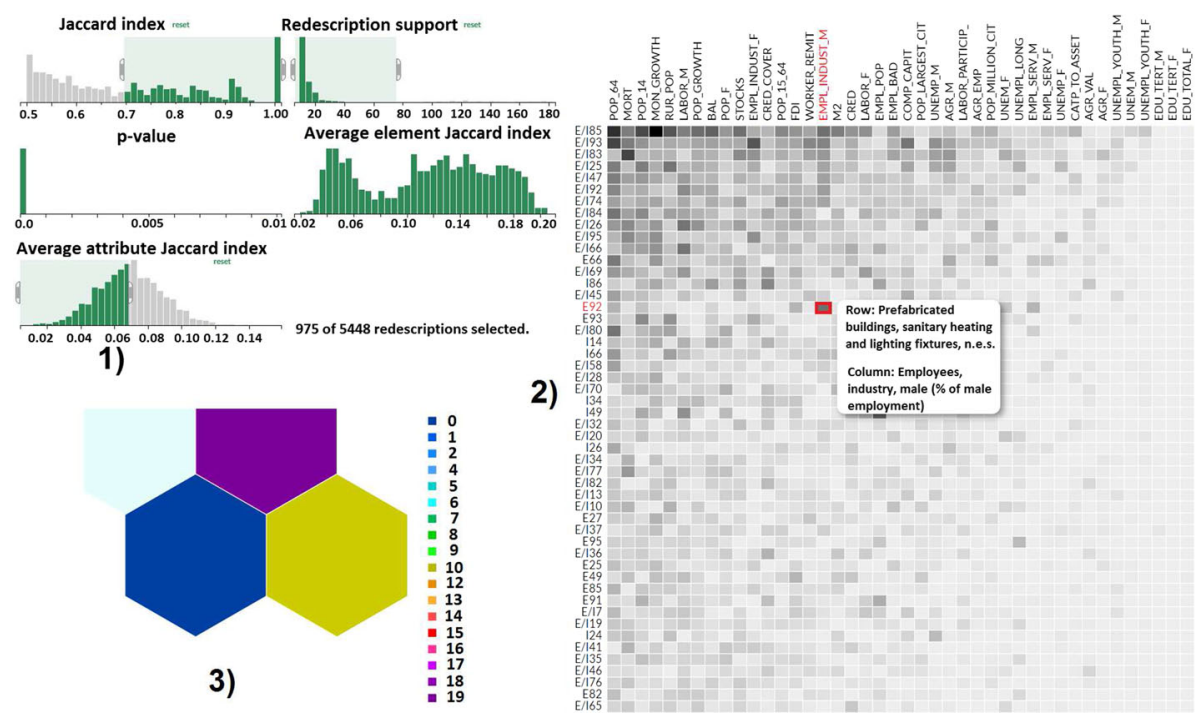

Fig. 8 Using interaction of different aspects to discover interesting knowledge. The number indicates the order in which different aspects were used

We started the analysis by using the property based aspect (see 1) in Fig. 8) to create the set $A=\mathcal{R}_{\mathcal{F}}$, where $\mathcal{F}=\{J \in[0.7,1.0]$, supp $\in[0,80], A A J \in[0,0.06]\}$. The goal was to select all highly accurate redescriptions that contain attributes that are not used that often in our redescription set (we used the attributes with average Jaccard contained in the lower half of the distribution). The support constraint was used to eliminate redescriptions describing majority of entities from the dataset because they are very general. Next, we used the attribute based exploration aspect to select all redescriptions that contain attributes a=export of prefabricated buildings, sanitary, heating, and lighting fixtures $\left(E_{92}\right)$ and $\mathrm{b}=$ employees industry male $(\%$ of population). We used the newly created set $A_{a, b}$ to create the SOM. The idea is to cluster the entities containing the selected two attributes and observe the similarities and or differences. The SOM map clustered the set $A_{a, b}$ into $A_{a, b}=C_{1} \cup C_{2} \cup C_{3} \cup C_{4}$, where $C_{3}=\emptyset$. We focus our analysis on two important clusters $C_{2}$ and $C_{4}$ with homogeneity 0.78 and 0.66 . The most important discovered property valid for all countries contained in the cluster $C_{2}$ is that $E_{92}=0$. The employment of males in industry matches the distribution of values for all countries contained in the dataset. As opposed to this cluster, countries contained in $C_{4}$ have $1 \leq E_{92} \leq 2$. Also, the employment of male population in industry significantly deviates (is higher) from the distribution of values of all countries.

\subsection{Exploring redescriptions obtained on the DBLP dataset}

In this section, we analyse the DBLP dataset, which significantly differs from the Country dataset. DBLP dataset differs largely from the Country dataset: it has much larger number of entities, it is very sparse and one view is a graph adjacency matrix. Authors have written a paper with smaller groups of other authors and mostly publish papers in conferences related to their research interests. As a result, redescriptions mostly have smaller support and lower accuracy. All attributes are Boolean, as opposed to numeric attributes contained in the Country dataset. 

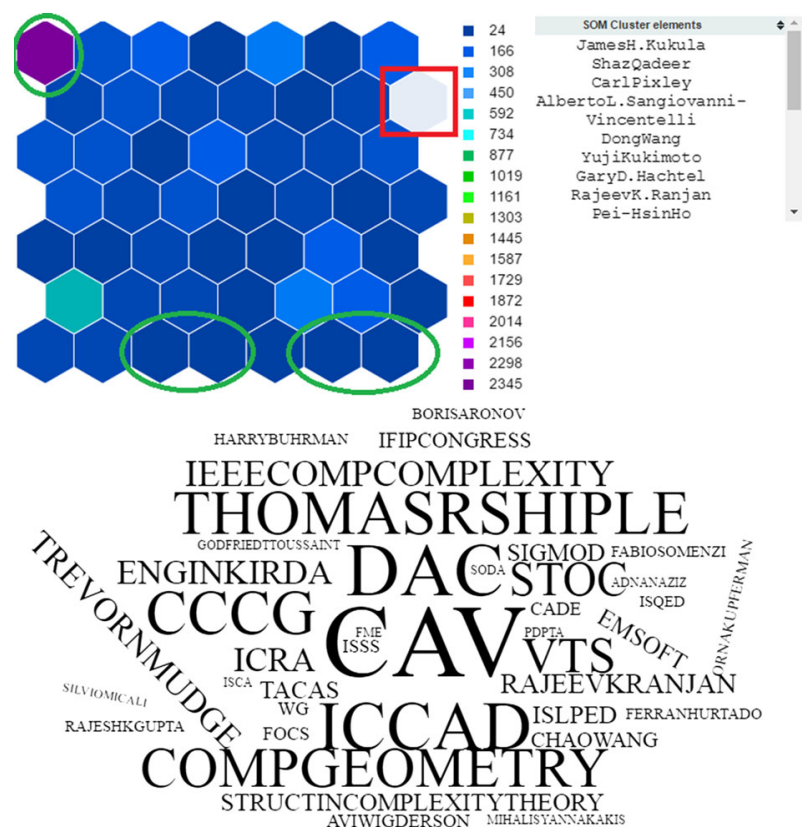

Fig. 9 SOM produced on the DBLP dataset

In the entity exploration aspect (presented in Fig. 9), we notice 6 clusters with homogeneity greater than 0.2 (circled in green and red). All encircled clusters, except the first one, contain less than 300 entities. The first encircled cluster, describes over 2000 entities and mostly contains redescriptions with very large support ( $>2000$ entities). Such redescriptions contain only negated variables and do not provide informative knowledge.

There exists 6 clusters with homogeneity large than 0.2 . Descriptions of these clusters can be found in Sect. 6.1 of Online resource 2.

We examine the cluster with maximal homogeneity of 0.4 (red square on Fig. 9). From the presented WordNet, we can see that the most occurring attributes in redescriptions contained in this cluster are Thomas R. Shiple, DAC, CAV, ICCAD and CompGeom. By examining the DBLP profile of Thomas R. Shiple, ${ }^{4}$ we can see that he published papers in DAC, CAV and ICCAD conferences, also his top 4 collaborators are: James H. Kukula, Adnan Aziz, Robert K. Brayton and Alberto L. Sangiovanni-Vincentelli. All four can be found in the selected cluster.

We present the most accurate redescription in Table 8.

This redescription describes around $40 \%$ of total co-authors of Thomas R. Shiple, all of which are members of the selected SOM cluster.

We perform similar analysis, using attribute based exploration, as in Mihelčić and Šmuc (2016). We arrange heatmap rows and columns by co-occurrence, compute the association score for all pairs of attributes in the top-left $5 \times 5$ submatrix and test the statistical significance of associations. We compute the Cramer's V (with correction), to determine the level of association between the pairs of Boolean attributes, and Pearson's chi-squared test to test the statistical significance of associations (formally, we perform the test of independence of two variables).

4 http://dblp.uni-trier.de. 
Table 8 Redescription $R_{\text {exd }}$

\begin{tabular}{ll}
\hline$R_{\text {exd }}:$ & $\left(q_{1_{\text {exd }}}, q_{2_{\text {exd }}}\right)$ \\
$q_{1_{\text {exd }}}:$ & $\neg \mathrm{ISSS} \wedge \mathrm{ICCAD} \wedge \neg \mathrm{CP} \wedge \neg \mathrm{ISQED} \wedge \neg \mathrm{VTS} \wedge \mathrm{CAV} \wedge \neg$ IFIPCongress \\
& $\wedge \mathrm{DATE}$ \\
$q_{2_{\text {exd }}}:$ & Thomas R. Shiple $\wedge \neg$ Trevor N. Mudge $\wedge \neg$ Orna Grumberg \\
& $\wedge \neg$ Freddy C. Mang \\
$\operatorname{supp}\left(R_{\text {exd }}\right)$ & Adnan Aziz, Fabio Somenzi, Felice Balarin, Gary D. Hachtel, James H. \\
& Kukula, Rajeev K. Ranjan, Robert K. Brayton, Sunil P. Khatri, Tiziano \\
& Villa, Vigyan Singhal, Yuji Kukimoto \\
& $J\left(R_{\text {exd }}=0.55\right), p\left(R_{\text {exd }}=0.0\right),\left|\operatorname{supp}\left(R_{\text {exd }}\right)\right|=11$ \\
\hline
\end{tabular}

Fig. 10 The $5 \times 5$ top-left submatrix of a heatmap produced on the DBLP dataset

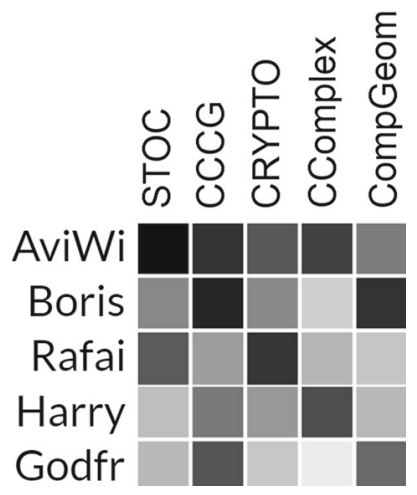

Table 9 Cramer's V score for a selected $5 \times 5$ cross-view attribute submatrix. For each attribute pair (below the association score), we display the $p$-value obtained by Pearson's chi-squared test, measuring the statistical significance of the association

\begin{tabular}{llllll}
\hline Cr. V & STOC & CCCG & CRYPTO & CComplex. & CompGeom \\
\hline A. Wigderson & 0.29 & 0.01 & 0.21 & 0.34 & 0.05 \\
& $<10^{-15}$ & 0.465 & $<10^{-15}$ & $<10^{-15}$ & $2.5 \cdot 10^{-4}$ \\
B. Aronov & 0.13 & 0.39 & 0.02 & 0.03 & 0.38 \\
& $<10^{-15}$ & $<10^{-15}$ & 0.19 & 0.046 & $<10^{-15}$ \\
R. Ostrovsky & 0.25 & 0.02 & 0.37 & 0.16 & 0.01 \\
& $<10^{-15}$ & 0.19 & $<10^{-15}$ & $<10^{-15}$ & 0.27 \\
H. Burhman & 0.13 & 0.01 & 0.07 & 0.36 & 0.01 \\
& $<10^{-15}$ & 0.31 & $2.9 \cdot 10^{-8}$ & $<10^{-15}$ & 0.71 \\
G. T. Toussaint & 0.04 & 0.46 & 0.004 & 0.01 & 0.37 \\
& 0.002 & $<10^{-15}$ & 0.78 & 0.3 & $<10^{-15}$ \\
\hline
\end{tabular}

The corresponding part of heatmap is presented in Fig. 10.

We can notice from Table 9 that the following pairs of attributes: (Avi Wigderson, CCCG), (Boris Aronov, CRYPTO), (Rafail Ostrovsky, CCCG), (Rafail Ostrovsky, CompGeom), (Harry Buhrman, CCCG), (Harry Buhrman, CompGeom), (Godfried T. Toussaint, CRYPTO), (Godfried T. Toussaint, CComplex) do not have statistically significant correla- 
Table 10 Redescriptions $R_{\text {start }}$ and $R_{\text {end }}$

\begin{tabular}{ll}
\hline$R_{\text {start }}:$ & $\left(q_{1_{\text {start }}}, q_{2_{\text {start }}}\right)$ \\
$q_{1_{\text {start }}}:$ & FOCS $\wedge$ CCCG $\wedge$ IEEEConferenceOnComputationalComplexity \\
$q_{2_{\text {start }}}:$ & Micha Sharir $\vee$ Harry Buhrman $\vee$ Ferran Hurtado $\vee$ Marc Joye $\vee$ \\
& Avi Wigderson \\
quality: & $J\left(R_{\text {start }}\right)=0.21, p\left(R_{\text {start }}\right)=0.0,\left|\operatorname{supp}\left(R_{\text {start }}\right)\right|=55$ \\
$R_{\text {end }}:$ & $\left(q_{1_{\text {end }}}, q_{2_{\text {end }}}\right)$ \\
$q_{1_{\text {end }}}:$ & EUROCRYPT $\wedge \neg$ ISTCS \\
$q_{2_{\text {end }}}:$ & Harry Buhrman $\vee$ Andrew B. Kahng $\vee$ Thomas R. Shiple $\vee$ David \\
& Pointcheval $\vee$ Bart Preneel $\vee$ Luca Trevisan $\vee$ Josef Pierpzyk $\vee$ \\
& Ran Canetti \\
quality: & $J\left(R_{\text {end }}\right)=0.21, p\left(R_{\text {end }}\right)=0,\left|\operatorname{supp}\left(R_{\text {end }}\right)\right|=66$ \\
\hline
\end{tabular}

tions. The higher co-occurrence of these pairs of attributes is a result of the attribute type (Boolean). The analysis of these pairs reveals that almost all co-authors of the stated authors never published a paper in the corresponding conferences. Thus, using the negated attribute corresponding to these conferences increases redescription accuracy (since all or majority of co-authors of a given author never published on this conference and many other non co-author authors that have published are removed from the query support set). This demonstrates that our approach can reveal many different types of associations (not necessarily only linear correlations) among one or more attributes.

From Table 9, we can see what are the research interests of the research communities to which the selected authors belong. By looking at the dblp profile of Avi Wigderson, we see that he published majority of papers in the STOC conference, IEEE Conference on Computational Complexity is also highly represented (as is for Harry Buhrman). Godfried T. Toussaint and Boris Aronov, published a number of papers in the CCCG conference, which connects them with authors doing research in computational geometry, Rafael Ostrovsky published a high number of papers in the CRYPTO and STOC conference.

We demonstrate the use of property based exploration aspect and redescription relation graph in a joined example. First, we use the crossfilter to remove all redescriptions with support larger than 2000 authors. In this way we ensure that all redescriptions containing only attributes with value false are removed from the redescription set. Next, we relate two redescriptions $R_{\text {start }}$ and $R_{\text {end }}$ with very small overlap (see Table 10 ).

$\operatorname{supp}\left(R_{\text {start }}\right) \cap \operatorname{supp}\left(R_{\text {end }}\right)=\{$ Miklosh Santha, Oded Goldreich, Omer Reingold, Ronen Shaltiel, Yevgeniy Dodis $\}$. The point of this analysis is to try to discover more connecting nodes or shared properties between these, apparently very loosely connected communities of authors using redescriptions contained in our redescription set. In the context of the presented use case, this information may be useful to authors performing research in both fields to locate potential collaborators, to find scientific papers of interest or potentially new and interesting scientific area for future research. Discovering such information is not a trivial task, and is hardly possible to achieve by simple analysis of individual redescriptions. In general, to perform this type of analysis, one has to use some sort of network analysis methodology (usually observing the interconnectivity between different detected communities in a network) in combination with various recommendation system algorithms, however those methods do not provide interpretative capabilities of our approach (especially in heterogeneous or multilayer networks). In order to achieve our goal, we set the minimal edge weight in the graph 
Fig. 11 Redescription relation graph obtained on the DBLP dataset

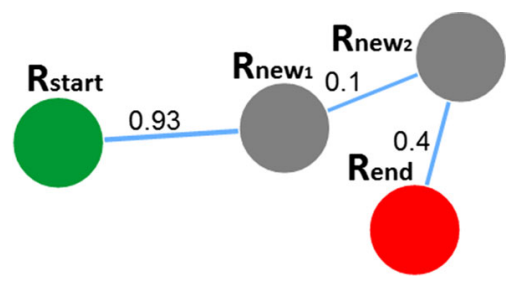

to 0.1 and create a redescription relation graph. The obtained relation graph is presented in Fig. 11.

The redescription $R_{\text {new }}$ is a small variation of the redescription $R_{\text {start }}\left(J\left(R_{\text {start }}, R_{\text {new } 1}\right)=\right.$ $0.93)$. $R_{\text {new }}$ has the same first query as the redescription $R_{\text {start }}$. The difference is in the second query: $q_{2_{\text {new }}}=$ Micha Sharir $\vee$ Harry Buhrman $\vee$ Marc Joye $\vee$ Avi Wigderson $\vee$ Godfried T. Toussaint $\vee$ Rosario Gennaro. $R_{n e w_{1}}$ describes 59 authors. Rosario Gennaro has mainly published papers on EUROCRYPT and CRYPTO but has also published two papers in the FOCS conference (he is a newly discovered link connecting FOCS and EUROCRYPT). Harry Buhrman, author that mainly publishes papers in the conferences related to complexity, also published one paper in the CRYPTO conference (which provides a link between FOCS and CRYPTO). Redescription $R_{n_{\text {ew }}}$ is very interesting and provides a link towards the redescription $R_{\text {end }}$. This redescription describes 67 authors that have published a paper in the CRYPTO conference and have not published a paper on Symphosium on Computational Geometry. Their co-author is at least one of the following authors: Micha Sharir, Harry Buhrman, Bart Preneel, Ran Caneti, Pierre McKenzie. There exist a total of 12 authors who satisfy the properties of both $R_{n e w_{1}}$ and $R_{n e w_{2}}$. Connection between redescriptions $R_{\text {new }_{2}}$ and $R_{\text {end }}$ is much higher $\left.\left(J\left(R_{\text {new }_{2}}, R_{\text {end }}\right)\right)=0.4\right)$ and the conferences CRYPTO and EUROCRYPT are thematically very similar. 38 authors share the properties defined in redescriptions $R_{\text {new }}$ and $R_{\text {end }}$.

In this example, we have observed two redescriptions, where one redescription described authors primarily publishing on FOCS and IEE Conference On Computational Complexity and the second primarily describing authors that publish papers on EUROCRYPT conference. Our analysis revealed two very important intermediary redescriptions that increase the level of connection and provide additional explanations for the relation between the two initially chosen redescriptions. Concretely, we have discovered a previously unknown author, Rosario Gennaro, that published papers both in EUROCRYPT and FOCS conferences, that there exist a conference called CRYPTO which shares large number of authors with the EUROCRYPT conference and that there exist authors (such as Harry Buhrman) that published papers on FOCS and CRYPTO conferences. As previously stated, such knowledge is extremely useful and not easy to find.

\subsection{Exploring redescriptions obtained on the phenotype dataset}

In this use case, we investigate associations between the occurrence of different COGs (Clusters of Orthologous Groups) of proteins (Tatusov et al. 1997, 2000) in a bacterial genome and bacterial phenotypes (the set of observable characteristics of an individual, created due to changes in a genome that were influenced by the environment). Phenotypic traits are represented as sets of words describing various bacterial characteristics. Detecting and understanding such connections is an interesting research problem in the field of biology (Brbić et al. 2016). 
The difference of our approach to that presented in Brbic et al. (2016) is that we use the unsupervised approach of redescription mining to relate sets of phenotypes to a set of COGs, whereas aforementioned work focused on detecting important genetic features for one selected phenotype (that was used as a target label), thus supervised approach was used to discover new and interesting knowledge. To reduce data size and sparsity, we only use the COGs and not the Unsupervised Clusters of Orthologous Group (NOG) (Powell et al. 2012).

On this use case, we discovered 9 different clusters of bacterial species with high average homogeneity $>0.5$ using entity-based exploration aspect. In this analysis, we focus on first two clusters (explained in this manuscript). Descriptions of all 9 clusters and a corresponding figure depicting obtained SOM can be seen in Sect. 6.2 of Online resource 2.

1. This cluster has average homogeneity 0.52 and contains 33 bacterial species. The most common phenotypic traits are: unicellular, photosystem, photosynthesis, ocean, fixat. From these phenotypes, we see that these bacteria are mostly unicellular capable of performing photosynthesis and that they mostly live in the ocean. The main representatives of this cluster are Synechococcus, Cyanothece and Nostoc genus of cyanobacteria.

2. The second cluster of interest has average homogeneity 0.71 and it contains 17 bacterial species. The most common discovered phenotypic traits are: haloarchaea, hypersalin, archaeal, seafood, $\mathrm{NaCl}$. The haloarchea (previously referred to as halobacteria), leave in the extreme environments (which is evident from the phenotype hypersalin) (Kennedy et al. 2001). This also explains the further two phenotypes seafood (since it is often found in salted fish) and $\mathrm{NaCl}$ because of the fact that high salinity attracts the haloarchea (Najjari et al. 2015).

Knowledge discovered by exploring the SOM map of the entity-based aspect brings very accurate grouping of different bacterial species contained in the data and reveals some of their interesting and distinguishing phenotypic properties. Such knowledge would be very hard to obtain by traversing a long list of redescriptions (which would needed to be done using Siren Galbrun and Miettinen (2012b)), on the other hand, using regular clustering would not reveal different properties of organisms discovered in our analyses. Similarities of cluster 4. and 5. (immediately noticeable from our SOM representation, since these are neighbouring clusters) may be completely overlooked if such mechanism is not used (for additional information see Sect. 6.2 of Online resource 2).

Next, we use the abilities of InterSet to observe interesting properties of bacterial genomes, for bacterial species contained in the first two clusters that may reveal environmental influence or specific adaptations that are reflected in bacterial phenotypes. To do this, we save a selected set of redescriptions (for each cluster in turn) and compute a heatmap (ordered by co-occurence) revealing interesting attribute associations for this specific selection.

Using redescriptions describing bacterial species contained in the first SOM cluster, we analysed attributes highly associated with phenotypic traits unicellular, photosystem, photosynthesis, ocean, fixat (attribute-pairs contained in the first column). We discovered the following interesting facts about the genomes of bacterial species contained in the first cluster:

1. There are several still uncharacterised clusters highly associated with the aforementioned phenotypic traits such as: $C O G_{5474}, C O G_{5135}, C O G_{3937}, C O G_{3330}$ etc. Discovery of their role and function is thus highly desirable.

2. $C_{5549}$ (predicted $\mathrm{Zn}$ - dependent protease), as has been shown in Jia et al. (2015) displays activity at optimal $\mathrm{pH} 8.0$ (which corresponds to the $\mathrm{pH}$ of sea water - the habitat of species contained in the first cluster). 
3. $C O G_{1149}$ is involved in DNA repair and is omnipresent in bacterial species. Thus, high association discovered is not surprising. Similar applies for $C O G_{4371}$ (predicted membrane protein).

4. $\mathrm{COG}_{74}$ (succinyl - CoA synthetase, alpha subunit). This gene cluster is used in phosphorylation (in aerobic metabolism functions). ${ }^{5}$ Phosporylation is performed by photosynthetic bacteria (Nozaki et al. 1961).

5. $C_{0} G_{5398}$ (heme oxygenase) is known to be a part in phytobilin biosynthesis (Cornejo et al. 1998). Phytobilin is a building unit of phycobiliproteins (that cause large fluorescence) which are mostly found in cyanobacteria. ${ }^{6}$

6. $C O G_{4100}$ (aluminum resistance). According to Fiore and Trevors (1994), cyanobacteria are often found in metal-contaminated fresh water habitats. Given this, a cluster of genes aimed at developing or providing aluminium resistance seems as inevitable adaptation (given usual toxicity of aluminium to life forms).

7. $C O G_{1360}$ (flagellar motion) is a common property in bacteria. The main aim of flagellar is to enable mobility of different bacterial species. $C O G_{4980}$ (gas vesicles protein) is an adaptation to the water environment which allows more efficient vertical migrations than by using flagella in water (Walsby 1994). This adaptation occurs in many different bacterial species.

Similar analysis performed on the second cluster by examining attributes associated to phenotipic traits (haloarchaea, hypersalin, archaeal, sea food, $\mathrm{NaCl}$ ) can be seen in Sect. 6.2 of Online resource 2.

Contextualization of redescriptions as provided by e.g. top $k$ co-occurring attributes or global associations in InterSet is not possible with Siren, as it does not focus on these characteristics of redescription sets.

Next, we present several interesting associations discovered with attribute-based aspect of InterSet, using all redescriptions created on the Phenotype dataset. We analyse the first five columns from top to bottom disregarding all uncharacterised COGs and pairs with low co-occurrence frequency and for each column present maximally two interesting findings. Cramer's V correlation score and statistical significance computed by Pearson's chi-squared test are presented in the sparse format $\left(a t_{1}, a t_{2}, \mathrm{Cr}\right.$. V, chi-square).

For the attribute shape coccus, we present (shape coccus, $C O G_{3351}, 0.28,<2.2 \cdot 10^{-16}$ ), (shape coccus, $\left.C O G_{1855}, 0.28,<2.2 \cdot 10^{-16}\right)$. $C O G_{3351}$, putative archaeal flagellar protein D/E, co-occurs with shape coccus in 73 different redescriptions. It has been shown in Desmond et al. (2007) that several different species of Pyrococcus, Thermococcus, Methanococcus and Methanocaldococcus contain clusters of genes connected with flagellum. Attribute $C O G_{1855}$ (ATPase - Pilt family) co-occurs with shape coccus in 59 redescriptions. It has been shown that in Myxococcus xanthus (Jakovljevic et al. 2008) the ATPase - Pilt family act as T4P extension/retraction cycle in vivo. Based on our findings, this gene cluster has a function in many different coccus-shaped bacterial species.

For the attribute (unicellular, photosystem, photosynthesi, ocean, fixat), we present ((unicellular, photosystem, photosynthesi, ocean, fixat), $\left.C O G_{4100}, 0.35,<2.2 \cdot 10^{-16}\right) . C O G_{4100}$ (Cystathionine beta-lyase family protein involved in aluminum resistance) co-occurs with the aforementioned attribute in 98 redescriptions. It has been shown that cyanobacteria can often be found in metal-contaminated fresh water habitats (Fiore and Trevors 1994). Thus, metal-resistance mechanisms are a must, especially for highly toxic metals as aluminium.

\footnotetext{
5 http://www.ebi.ac.uk/interpro/entry/IPR005810.

6 https://www.sciencedirect.com/topics/agricultural-and-biological-sciences/phycobiliprotein.
} 
Fig. 12 Redescription relation graph obtained on the Phenotype dataset relating redescriptions $R_{b s}$ and $R_{b e}$

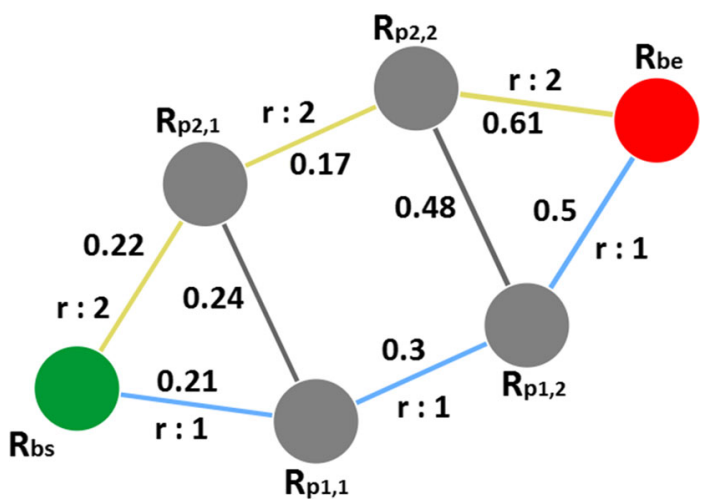

Metabolism PAH degrading attribute co-occurs in 59 redescriptions with attribute $C_{1218}$ (3-Phosphoadenosine 5-phoshosulfate (PAPS) 3-phosphatase). This pair of attributes has statistically significant correlation: (metabolism PAH degrading, COG 1218 , $0.13,5.8 \cdot 10^{-6}$ ). Research into whether this group of genes is more frequently occurring in PAH degrading bacteria and its potential role in the process is needed to assess the relevance of this association.

Oxygen requirement strict anaero co-occurs in 65 redescriptions with attribute $C O G_{1592}$ (Rubrerythrin). The correlation between these two attributes is statistically significant (metabolism PAH degrading, $C O G_{1592}, 0.72,<2.2 \cdot 10^{-16}$ ). The usage of rubrerythrin in anaerob bacteria has been confirmed (Jenney et al. 1999; Lumppio et al. 2001; Jennings et al. 2014) in the process of degrading $\mathrm{O}_{2}^{-}$and $\mathrm{H}_{2} \mathrm{O}_{2}$ without producing $\mathrm{O}_{2}$.

The attribute (hyperthermophil, archaeal, hydrotherm, vent, heterotroph), is significantly associated (co-occurring in 37 redescriptions) and correlated with attribute $C O G_{3351}$ (putative archaeal flagellar protein D/E), ((hyperthermophil, archaeal, hydrotherm, vent, heterotroph), $\left.C O G_{3351}, 0.49,<2.2 \cdot 10^{-16}\right)$. This attribute shares many phenotypic traits with those observed on species from cluster 9 (containing exclusively species from genus coccus).

Next, we attempt to find interesting, shared properties and relations between hyperthermophil non-coccus bacteria and coccus bacteria with the phenotypic traits (hyperthermophil, archaeal, hydrotherm, vent, heterotroph).

This might be possible using heatmap and analysing the obtained subset of redescriptions. However, this has two main disadvantages: a) it restricts the subset of redescriptions to those containing a selected pair of attributes, b) user has to traverse the list and analyse many uninteresting redescriptions (e.g those containing exclusively bacterial species from genus coccus).

Because of this we use redescription relation graph to create top 2 shortest paths connecting redescriptions $R_{b s}$ and $R_{b e}$. Redescription $R_{b s}$ describes bacteria living in hot springs which do not belong to genus coccus whereas redescription $R_{b e}$ describes bacterial species belonging to genus coccus that contain phenotypic properties (hyperthermophil, archaeal, hydrotherm, vent, heterotroph). Definitions and properties of a starting, ending and all newly discovered redescriptions are available in Table 11. Redescriptions contained in the first path are denoted $R_{p_{1, i}}$, analogously redescriptions contained on the second path are denoted $R_{p_{2, i}}$. The obtained graph can be seen in Fig. 12.

Descriptions of all $C O G$ s used in Table 11 are available in Table 12.

Redescription $R_{b s}$ describes 12 different non-coccus bacteria that inhabit hot springs and are by their nature thermophiles. Redescription $R_{p_{1,1}}$ describes hyperthermophilic 
Table 11 Redescriptions contained in a redescription relation graph starting from $R_{b s}$ and ending at $R_{b e}$

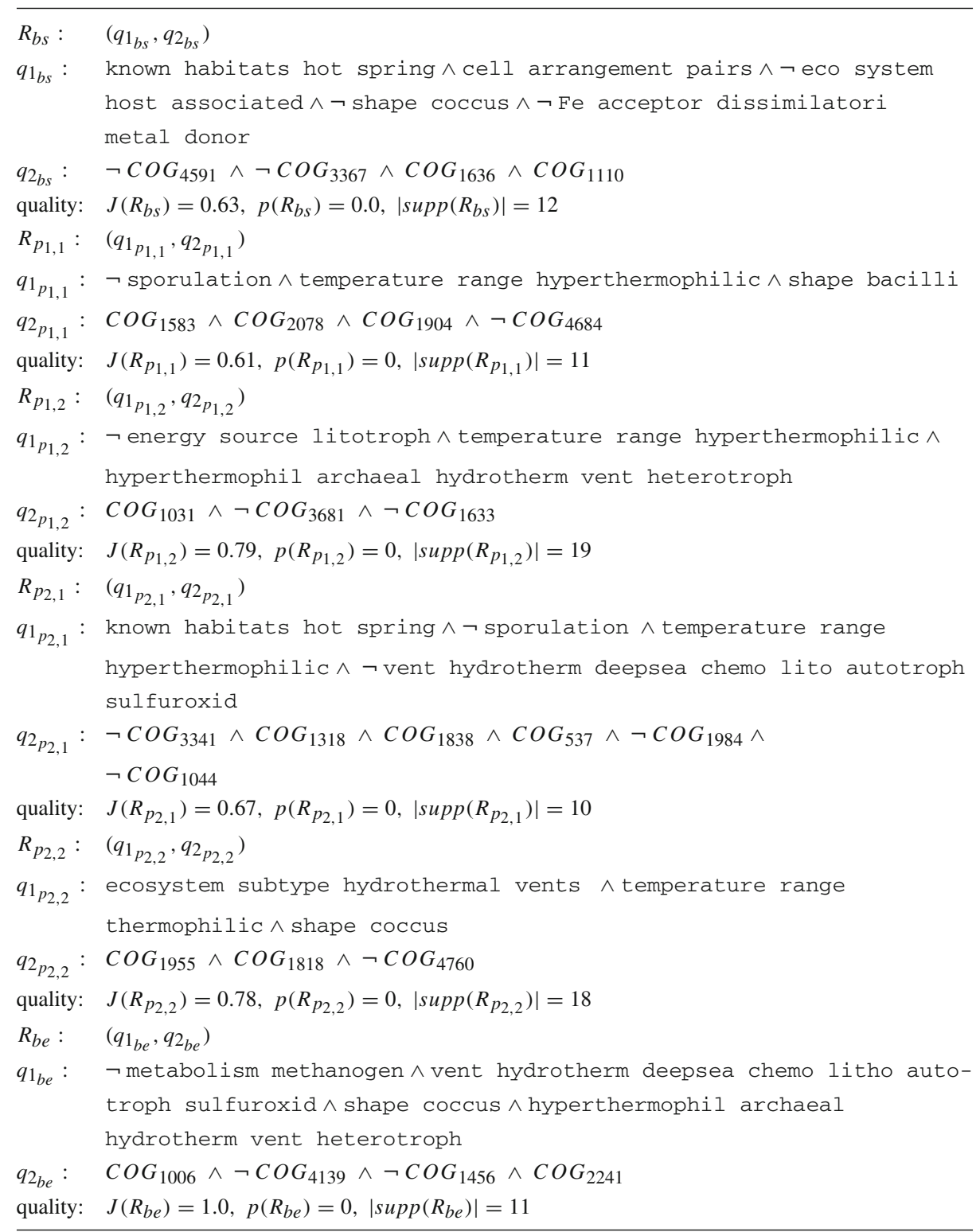

bacterial species with a rod-shape (called bacilli). Bacterial species Caldicellulosiruptor hydrothermalis, Caldicellulosiruptor kristjanssonii, Caldicellulosiruptor obsidiansis and Caldicellulosiruptor owensensis have properties described by both $R_{b s}$ and $R_{p_{1,1}}$. They share properties described by $R_{p_{1,1}}$ with bacterial species Thermotoga maritima, Thermotoga naphthophila, Thermotoga neapolitana, Thermotoga petrophila and Thermotoga sp. RQ2. These species also satisfy the properties described by redescription $R_{p_{1,2}}$, in particular (hyperthermophil, archaeal, hydrotherm, vent, heterotroph). They share these properties with a number 
Table 12 Descriptions of attributes occurring in redescriptions $R_{\text {start }}, R_{i_{1}}$ and $R_{\text {end }}$

\begin{tabular}{|c|c|}
\hline Attribute & Description \\
\hline $\mathrm{COG}_{537}$ & $\begin{array}{l}\text { Diadenosine tetraphosphate (Ap4A) hydrolase and other HIT family } \\
\text { hydrolases }\end{array}$ \\
\hline$C O G_{1006}$ & Multisubunit $\mathrm{Na}+/ \mathrm{H}+$ antiporter, MnhC subunit \\
\hline$C O G_{1031}$ & Uncharacterized Fe-S oxidoreductase \\
\hline$C O G_{1044}$ & UDP-3-O-[3-hydroxymyristoyl] glucosamine $\mathrm{N}$-acyltransferase \\
\hline$C O G_{1110}$ & Reverse gyrase \\
\hline$C O G_{1318}$ & Predicted transcriptional regulators \\
\hline$C O G_{1456}$ & $\begin{array}{l}\mathrm{CO} \text { dehydrogenase/acetyl-CoA synthase gamma subunit (corrinoid Fe-S } \\
\text { protein) }\end{array}$ \\
\hline$C O G_{1583}$ & $\begin{array}{l}\text { Uncharacterized protein predicted to be involved in DNA repair (RAMP } \\
\text { superfamily) }\end{array}$ \\
\hline$C O G_{1633}$ & Uncharacterized conserved protein \\
\hline$C O G_{1636}$ & Uncharacterized protein conserved in bacteria \\
\hline$C O G_{1818}$ & Predicted RNA-binding protein, contains THUMP domain \\
\hline$C O G_{1838}$ & $\begin{array}{l}\text { Tartrate dehydratase beta subunit/Fumarate hydratase class I, C-terminal } \\
\text { domain }\end{array}$ \\
\hline$C O G_{1904}$ & Glucuronate isomerase \\
\hline$C O G_{1955}$ & Archaeal flagella assembly protein $\mathrm{J}$ \\
\hline$C O G_{1984}$ & Allophanate hydrolase subunit 2 \\
\hline$C O G_{2078}$ & Uncharacterized conserved protein \\
\hline$C O G_{2441}$ & Predicted butyrate kinase \\
\hline$C O G_{3341}$ & Predicted double-stranded RNA/RNA-DNA hybrid binding protein \\
\hline$C O G_{3367}$ & Uncharacterized conserved protein \\
\hline$C O G_{3681}$ & Uncharacterized conserved protein \\
\hline$C O G_{4139}$ & ABC-type cobalamin transport system, permease component \\
\hline $\mathrm{COG}_{4591}$ & $\begin{array}{l}\text { ABC-type transport system, involved in lipoprotein release, permease } \\
\text { component }\end{array}$ \\
\hline $\mathrm{COG}_{4684}$ & Predicted membrane protein \\
\hline$C O G_{4760}$ & Predicted membrane protein \\
\hline
\end{tabular}

of coccus bacteria from genus Pyrococcus and Thermococcus. All coccus bacterial species, with the exception of Pyrococcus furiosus and Thermococcus barophilus are described by the redescription $R_{b e}$.

To summarize, starting from a pair of redescriptions with completely disjoint support sets, describing bacteria of different shapes (coccus and non-coccus), we have managed to find shared phenotipic and genetic properties between coccus bacterial species and rod-shaped bacilli species. In addition, we located the exact bacterial species from both the coccus and bacilli group that share these common properties.

Discovering such knowledge using attribute-based aspect would be difficult, since it would require potentially traversing many different redescriptions from the selected subset. In addition, it would require using the external sources of information to determine the type and level of connection between different redescriptions. 
Table 13 Execution times of different requests and algorithms for C - country, D - DBLP and P - Phenotype data set used in this study

\begin{tabular}{lllllllll}
\hline Data & Init-L & SOM-L & Heat-L & RedA-L & EntJ-L & SOM-A & Filt-A & RRG-A \\
\hline$C$ & $4.5 \mathrm{~s}$ & $1.12 \mathrm{~s}$ & $7 \mathrm{~s}$ & $140 \mathrm{~ms}$ & $5.2 \mathrm{~m}$ & $4.2 \mathrm{~m}$ & $3.7 \mathrm{~s}$ & $2.2 \mathrm{~m}$ \\
$D$ & $16.3 \mathrm{~s}$ & $3.36 \mathrm{~s}$ & $7 \mathrm{~s}$ & $2 \mathrm{~s}$ & $2.5 \mathrm{~m}$ & $81 \mathrm{~m}$ & $1.6 \mathrm{~s}$ & $0.2 \mathrm{~s}$ \\
$P$ & $2 \mathrm{~s}$ & $762 \mathrm{~ms}$ & $15 \mathrm{~s}$ & $200 \mathrm{~ms}$ & $7.1 \mathrm{~m}$ & $31 \mathrm{~m}$ & $4.6 \mathrm{~s}$ & $11.8 \mathrm{~s}$ \\
\hline
\end{tabular}

\section{InterSet time complexity considerations}

InterSet is a web-based application consisting of a set of algorithms and tools designed to improve user's understanding of various problems analysed with redescription mining. Assessing the true execution time is difficult since it depends not only on the complexity of algorithms contained within it, but also on the type of database and storage used, server load, network connectivity and many others.

\subsection{Time complexity analysis of InterSet}

A detailed analysis of computational complexity of the InterSet tool is available in Sect. 7 of Online resource 2. It can be seen that InterSet computational complexity depends on number of redescriptions contained in the redescription set, number of entities and number of attributes contained in the input data. Currently developed tool was tested on redescription sets containing maximally 6200 redescriptions. Enabling exploration of extremely large redescription sets, these containing $>10000$ redescriptions would require examining and solving various different technical challenges arising in HTML, JavaScript algorithmic implementations and currently used type of database.

\subsection{Empirical evaluation of InterSet}

As can be seen from Sect. 7 from Online resource 2, obtaining all redescription entity/attribute Jaccard indexes is computationally demanding. We optimised the corresponding database query that loads this data by adding index on the first column representing redescription id of a first redescription in the pair. Since data loading of this size currently needs to be done in chunks, adding this index allows searching each of $\frac{|\mathcal{R}|^{2}}{k}$ pairs of redescriptions using logarithmic search. By adding index on the column representing one redescription id, we empirically get between $20 \%-30 \%$ execution speed-up.

Execution times of various data requests and algorithm executions, obtained on a fast internet connection, using Goggle Chrome browser (in Incognito mode) and a Windows 10 PC with Intel Core i $74770 \mathrm{CPU}(3.4 \mathrm{GHz})$ with $16 \mathrm{~Gb}$ of RAM memory are available in Table 13. $L$ denotes load time and $A$ algorithm execution time whereas Init denotes initialization data (performed only once), $S O M-L$ denotes loading SOM layout, Heat loading heatmap, RedA loading data required for individual redescription analysis, EntJ loading entity Jaccard data (case with attributes is equivalent), SOM-A denotes constructing a SOM map (without loading the graph from database) on a whole redescription set $\mathcal{R}$ (using default settings $4 \times 4$ layout, learning rate of 0.1 and $|E|$ iterations, achieved by using number of iterations $=a l l)$, Filt denotes filtering the redescription set $\mathcal{R}(\max$ entity/attribute Jaccard 


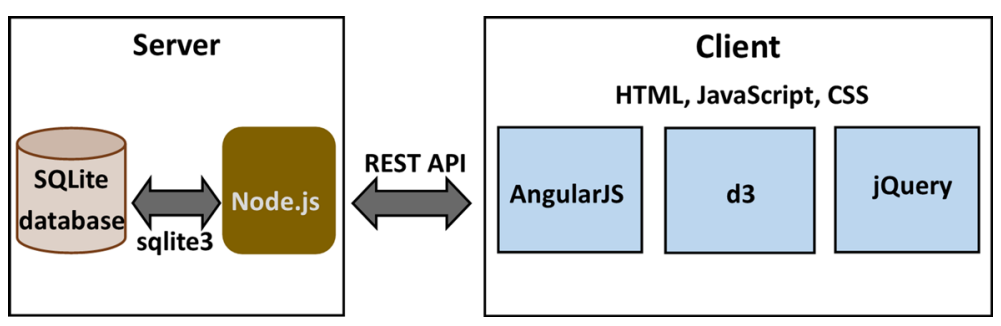

Fig. 13 Architecture of the InterSet application

of 0.1 was used, without loading the entity/attribute Jaccard data) and RRG denotes creating and drawing a redescription relation graph using redescriptions from $\mathcal{R}$ (we construct two paths containing a starting, ending and one intermediary node with minimal edge weight of 0.1 ). Loading time of entity Jaccard data is not included in this analysis.

\section{Implementation details}

The InterSet tool is a web-based application created using the HTML, ${ }^{7} \mathrm{JavaScript}^{8}$ and CSS. ${ }^{9}$ It consists of a client interface, created as an AngularJS application ${ }^{10}$ which we use in combination with the D3. j s library ${ }^{11}$ and the JQuery ${ }^{12}$ along with several visualizations developed in them to allow better data representation and manipulation. The underlying data is saved in a SQLite database ${ }^{13}$ and the communication between the database and the client is achieved with the Node.js runtime. ${ }^{14}$ The Node.js and the SQLite constitute the server-side of the application (for the entire architecture see Fig. 13).

As a consequence of using AngularJs and Node.js, the tool is optimized to work on Google Chrome browser ${ }^{15}$ (64-bit version needed for full functionality), however it also works on other popular browsers (e.g. Mozzila Firefox ${ }^{16}$ and Internet Explorer ${ }^{17}$ ) though the 32-bit versions might experience memory problems when creating redescription relation graphs. All the resources used to make the tool along with the instructions on how to set it up and use it are available on the tool demo homepage. ${ }^{18}$ The source code of the InterSet tool ${ }^{19}$ and the source code of the CLUS-RM algorithm ${ }^{20}$ used to create a redescription set are available on GitHub.

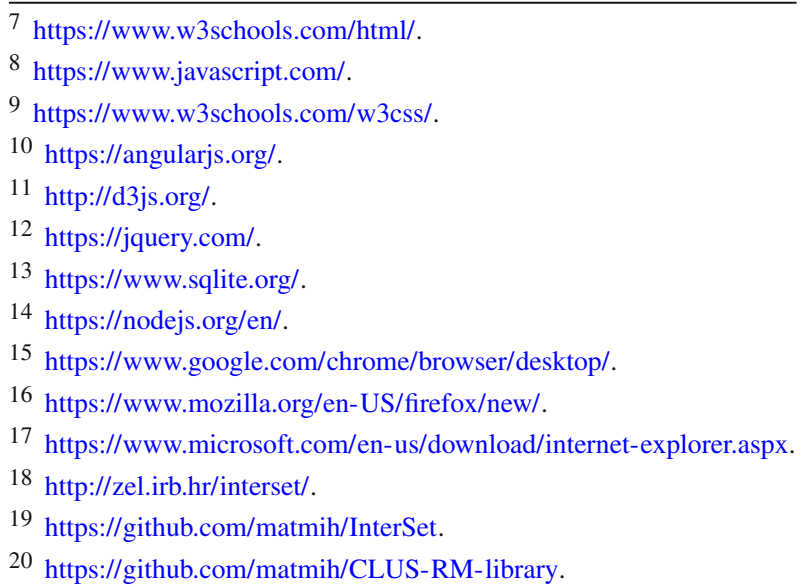




\section{Conclusion and future work}

In this work, we presented the InterSet tool that allows targeted and contextual exploration of redescription sets. The tool provides various types of analysis at different levels of granularity: at the level of a redescription set, interaction between smaller groups of redescriptions or at the level of individual redescriptions. The tool is designed to help researchers in two important steps of sensemaking (Endert et al. 2014a, b): (i) search for evidence and relations, (ii) building and searching evidence for hypothesis. In addition, it is naturally suited to allow provenance (Alspaugh et al. 2014; Ragan et al. 2016; Guo et al. 2016). There are several properties of InterSet that support the integration of provenance: a) its use of a database, b) existence of functions for storing/loading different representations of visualizations to a database and c) existence of a system for logging users. Using these already developed components, the tool can be extended to keep track of every user action, making research transparent and fully reproducible. We have demonstrated the usefulness of the exploration techniques enabled by the InterSet on three different use case datasets. Redescriptions obtained on the Country dataset describe world countries using two disjoint sets of numerical attributes. Although small in size, this dataset allows discovering large number of highly accurate redescriptions. It is substantially harder to obtain redescriptions of similar accuracy on a much larger dataset DBLP, which is our second use case dataset. Despite this, using our exploration techniques, we managed to find very interesting knowledge. Exploration of redescriptions obtained on a Phenotype dataset, which re-describe a set of bacterial species using information about their COGs and phenotypic traits, revealed many interesting and scientifically verified facts, but also pointed out COG examples whose function is still unknown and potentially interesting for further scientific study.

Distinct capabilities of the InterSet such as contextualization, targeted exploration and discovery of redescription relations, that all stem from the focus on dealing with redescription sets, not only individual redescriptions, make it unique as an interactive exploration tool in the field of redscription mining.

In future work, we intend to extend the applicability of the tool for exploration of other kinds of rule sets, such as association rules and subgroups. The tool will also be extended to work with different redescription set construction techniques (Kalofolias et al. 2016; Mihelčić et al. 2017a), and enable the use of statistical tests within different aspects to facilitate the discovery process. One potentially interesting direction of future research is to explore the potential of developed algorithms and adapted visualizations in an interactive redescription mining setting.

We also plan to experiment with different types of databases (or combinations thereof) for storing data. The main goal is to reduce the response time of the application, which is now relying on the more time consuming communication with relational database.

Acknowledgements The authors acknowledge the European Commission's support through the MAESTRA project (Gr. No. 612944), support of the Croatian Science Foundation (Pr. No. 9623: Machine Learning Algorithms for Insightful Analysis of Complex Data Structures) and partial support by the Centre of Excellence project "DATACROSS", co-financed by the Croatian Government and the European Union through the European Regional Development Fund - the Competitiveness and Cohesion Operational Programme (KK.01.1.1.01.0009). 


\section{References}

Alspaugh, S., Ganapathi, A., Hearst, M.A., \& Katz, R. (2014). Better logging to improve interactive data analysis tools. In $K D D$ workshop on interactive data exploration and analytics (IDEA).

Andrienko, G., \& Andrienko, N. (1999). Interactive maps for visual data exploration. International Journal of Geographical Information Science, 13(4), 355-374.

Appice, A., \& Buono, P. (2005). Analyzing multi-level spatial association rules through a graph-based visualization. In IEA/AIE, Springer, lecture notes in computer science, vol. 3533, pp. 448-458.

Berthold, M. R., Cebron, N., Dill, F., Gabriel, T. R., Kötter, T., Meinl, T., et al. (2009). Knime-the konstanz information miner: Version 2.0 and beyond. SIGKDD Explorations Newsletter, 11(1), $26-31$.

Blanchard, J., Guillet, F., \& Briand, H. (2003). A user-driven and quality-oriented visualization for mining association rules. In Proceedings of the 3rd IEEE international conference on data mining (ICDM), Melbourne, Florida, USA, pp. 493-496

Boley, M., Mampaey, M., Kang, B., Tokmakov, P., \& Wrobel, S. (2013). One click mining: Interactive local pattern discovery through implicit preference and performance learning. In Proceedings of the ACM SIGKDD workshop on interactive data exploration and analytics. ACM, New York, NY, USA, IDEA '13, pp. 27-35

Brbić, M., Piškorec, M., Vidulin, V., Kriško, A., Šmuc, T., \& Supek, F. (2016). The landscape of microbial phenotypic traits and associated genes. Nucleic Acids Research, 44(21), 10,074-10,090.

Castillo-Rojas, W., Peralta, A., \& Meneses, C. (2014). Augmented visualization of association rules for data mining. In Eight Alberto Mendelzon workshop on foundations of data management, Cartagena de Indias, Colombia, $A M W^{\prime} 14$.

Chakravarthy, S., Zhang, H. (2003). Visualization of association rules over relational DBMSs. In Proceedings of the 2003 ACM symposium on applied computing, ACM, New York, NY, USA, SAC '03, pp. 922-926.

Chau, DH., Kittur, A., Hong, JI., \& Faloutsos, C. (2011). Apolo: Making sense of large network data by combining rich user interaction and machine learning. In Proceedings of the SIGCHI conference on human factors in computing systems. ACM, New York, NY, USA, CHI '11, pp. 167-176.

Chau, DH., Akoglu, L., Vreeken, J., Tong, H., \& Faloutsos, C. (2012). Tourviz: Interactive visualization of connection pathways in large graphs. In Proceedings of the 18th ACM SIGKDD international conference on knowledge discovery and data mining, ACM, New York, NY, USA, KDD '12, pp. 1516-1519.

Cornejo, J., Willows, R. D., \& Beale, S. I. (1998). Phytobilin biosynthesis: cloning and expression of a gene encoding soluble ferredoxin-dependent heme oxygenase from synechocystis sp. pcc 6803. The Plant Journal, 15(1), 99-107.

DBLP (2010) DBLP dataset. http://dblp.uni-trier.de/db

De Bie, T., Kontonasios, K. N., \& Spyropoulou, E. (2010). A framework for mining interesting pattern sets. In Proceedings of the ACM SIGKDD workshop on useful patterns. ACM, New York, NY, USA, UP '10, pp. 27-35.

De Raedt, L., Nijssen, S., \& Guns, T. (2013). k-pattern set mining under constraints. IEEE Transactions on Knowledge \& Data Engineering, 25, 402-418.

Desmond, E., Brochier-Armanet, C., \& Gribaldo, S. (2007). Phylogenomics of the archaeal flagellum: rare horizontal gene transfer in a unique motility structure. BMC Evolutionary Biology, 7(1), 106.

Dijkstra, E. W. (1959). A note on two problems in connexion with graphs. Numerische Mathematik, 1(1), 269-271.

Endert, A., Hossain, M. S., Ramakrishnan, N., North, C., Fiaux, P., \& Andrews, C. (2014a). The human is the loop: New directions for visual analytics. Journal of Intelligent Information Systems, 43(3), 411-435.

Endert, A., North, C., Chang, R., \& Zhou, M. (2014b). Toward usable interactive analytics: Coupling cognition and computation. In: Proceedings of the 2014 Workshop on Interactive Data Exploration and Analytics at KDD (IDEA)

Fiore, M., \& Trevors, J. (1994). Cell composition and metal tolerance in cyanobacteria. Biometals, 7(2), 83-103.

Galbrun, E. (2013). Methods for Redescription Mining. PhD thesis, University of Helsinki, Finland

Galbrun, E., \& Miettinen, P. (2012a). From black and white to full color: Extending redescription mining outside the boolean world. Statistical Analysis and Data Mining, 5(4), 284-303.

Galbrun, E., \& Miettinen, P. (2012b). Siren: An interactive tool for mining and visualizing geospatial redescriptions. In Proceedings of the 18th ACM SIGKDD international conference on knowledge discovery and data mining, ACM, New York, NY, USA, KDD '12, pp. 1544-1547.

Galbrun, E., \& Miettinen, P. (2016). Mining redescriptions with siren. ACM Transactions on Knowledge Discovery from Data: In Press. 
Gallo, A., Miettinen, P., \& Mannila, H. (2008). Finding subgroups having several descriptions: Algorithms for redescription mining. In Proceedings of the SIAM international conference on data mining (SDM), SIAM, pp. 334-345.

Gamberger, D., Mihelčić, M., \& Lavrač, N. (2014), Multilayer clustering: A discovery experiment on country level trading data. In Proceedings of the 17th international conference discovery science, DS 2014, Bled, Slovenia, pp 87-98.

Goethals, B., Moens, S., \& Vreeken, J. (2011). Mime: A framework for interactive visual pattern mining. In Proceedings of the 17th ACM SIGKDD international conference on knowledge discovery and data mining, ACM, New York, NY, USA, KDD '11, pp. 757-760.

Guo, H., Gomez, S. R., Ziemkiewicz, C., \& Laidlaw, D. H. (2016). A case study using visualization interaction logs and insight metrics to understand how analysts arrive at insights. IEEE Transactions on Visualization and Computer Graphics, 22(1), 51-60.

Hofmann, M., \& Klinkenberg, R. (2013). RapidMiner: Data mining use cases and business analytics applications. London: Chapman \& Hall/CRC.

Jakovljevic, V., Leonardy, S., Hoppert, M., \& Søgaard-Andersen, L. (2008). Pilb and pilt are atpases acting antagonistically in type iv pilus function in myxococcus xanthus. Journal of Bacteriology, 190(7), 24112421.

Jenney, F. E., Verhagen, M. F., Cui, X., \& Adams, M. W. (1999). Anaerobic microbes: Oxygen detoxification without superoxide dismutase. Science, 286(5438), 306-309.

Jennings, M. E., Schaff, C. W., Horne, A. J., Lessner, F. H., \& Lessner, D. J. (2014). Expression of a bacterial catalase in a strictly anaerobic methanogen significantly increases tolerance to hydrogen peroxide but not oxygen. Microbiology, 160(2), 270-278.

Jia, B., Li, Z., Liu, J., Sun, Y., Jia, X., Xuan, Y. H., et al. (2015). A zinc-dependent protease amz-tk from a thermophilic archaeon is a new member of the archaemetzincin protein family. Frontiers in Microbiology, 6,1380 .

Kalofolias, J., Galbrun, E., \& Miettinen, P. (2016). From sets of good redescriptions to good sets of redescriptions. In Proceedings of the 16th IEEE international conference on data mining (ICDM'16). IEEE, Los Alamitos. To appear.

Kennedy, S. P., Ng, W. V., Salzberg, S. L., Hood, L., \& DasSarma, S. (2001). Understanding the adaptation of halobacterium species nrc-1 to its extreme environment through computational analysis of its genome sequence. Genome Research, 11(10), 1641-1650.

Knobbe, A. J., \& Ho, E. K. Y. (2006). Pattern teams (pp. 577-584). Berlin, Heidelberg: Springer.

Kohonen, T., Schroeder, R. M., \& Huang, T. S. T. (Eds.). (2001). Self-organizing maps (3rd ed.). USA: Springer, New York Inc.

Kranjc, J., Podpecan, V., \& Lavrac, N. (2012) Clowdflows: A cloud based scientific workflow platform. In Flach PA, Bie TD, Cristianini N (Eds.) ECML/PKDD (2), Springer, Lecture Notes in Computer Science, vol. 7524, pp. 816-819.

Kroening, D., \& Strichman, O. (2008). Decision procedures: An algorithmic point of view (1st ed.). Incorporated: Springer Publishing Company.

Kumar, D., Ramakrishnan, N., Helm, R. F., \& Potts, M. (2008). Algorithms for storytelling. IEEE Transactions on Knowledge and Data Engineering, 20(6), 736-751.

Lam, H. T., Mörchen, F., Fradkin, D., \& Calders, T. (2014). Mining compressing sequential patterns. Statistical Analysis and Data Mining, 7(1), 34-52.

Liu, G., Suchitra, A., Zhang, H., Feng, M., Ng, S. K., \& Wong, L. (2012). Assocexplorer: An association rule visualization system for exploratory data analysis. In $K D D, A C M$, pp. 1536-1539.

Lumppio, H. L., Shenvi, N. V., Summers, A. O., Voordouw, G., \& Kurtz, D. M. (2001). Rubrerythrin and rubredoxin oxidoreductase indesulfovibrio vulgaris: A novel oxidative stress protection system. Journal of Bacteriology, 183(1), 101-108.

Michael, H., Sudheer, C., Kurt, H., \& Christian, B. (2011). The arules R-package ecosystem: Analyzing interesting patterns from large transaction data sets. Journal of Machine Learning Research, 12, 20212025.

Miettinen, P. (2014). Interactive data mining considered harmful (if done wrong). In ACM SIGKDD 2014 full-day workshop on interactive data exploration and analytics, pp. 85-87.

Mihelcic, M., Dzeroski, S., Lavrac, N., \& Smuc, T. (2018). Redescription mining augmented with random forest of multi-target predictive clustering trees. Journal of Intelligent Information Systems, 50(1), 63-96.

Mihelčić, M., \& Šmuc, T. (2016) Interset: Interactive redescription set exploration. In Discovery Science 19th international conference, DS 2016, Bari, Italy, October 19-21, 2016, Proceedings, pp. 35-50.

Mihelčić, M., Džeroski, S., Lavrać, N., \& Šmuc, T. (2015). Redescription mining with multi-target predictive clustering trees. In New frontiers in mining complex patterns - 4th international workshop (pp. 125-143). Porto, Portugal: NFMCP. 
Mihelčić, M., Džeroski, S., Lavrač, N., \& Šmuc, T. (2017a). A framework for redescription set construction. Expert Systems with Applications, 68, 196-215.

Mihelčić, M., Šimić, G., Babić Leko, M., Lavrač, N., Džeroski, S., Šmuc, T., et al. (2017b). Using redescription mining to relate clinical and biological characteristics of cognitively impaired and alzheimer's disease patients. PLOS ONE, 12(10), 1-35.

Najjari, A., Elshahed, M. S., Cherif, A., \& Youssef, N. H. (2015). Patterns and determinants of halophilic archaea (class halobacteria) diversity in tunisian endorheic salt lakes and sebkhet systems. Applied and Environmental Microbiology, 81(13), 4432-4441.

Nozaki, M., Tagawa, K., \& Arnon, D. I. (1961). Noncyclic photophosphorylation in photosynthetic bacteria. Proceedings of the National Academy of Sciences, 47(9), 1334-1340.

Ouali, A., Zimmermann, A., Loudni, S., Lebbah, Y., Crémilleux, B., Boizumault, P., \& Loukil, L. (2017). Integer Linear Programming for Pattern Set Mining; with an Application to Tiling. In Pacific-Asia Conference on Knowledge Discovery and Data Mining, Jeju, South Korea, Advances in Knowledge Discovery and Data Mining 21st Pacific-Asia Conference, PAKDD 2017, Jeju, South Korea, May 23-26, 2017, Proceedings.

Parida, L., \& Ramakrishnan, N. (2005), Redescription mining: Structure theory and algorithms. In $A A A I, A A A I$ Press / The MIT Press, pp. 837-844.

Pei, J., Han, J., \& Wang, W. (2007). Constraint-based sequential pattern mining: The pattern-growth methods. Journal of Intelligent Information Systems, 28(2), 133-160.

Powell, S., Szklarczyk, D., Trachana, K., Roth, A., Kuhn, M., Muller, J., et al. (2012). eggnog v3.0: Orthologous groups covering 1133 organisms at 41 different taxonomic ranges. Nucleic Acids Research, 40(D1), D284-D289.

Ragan, E. D., Endert, A., Sanyal, J., \& Chen, J. (2016). Characterizing provenance in visualization and data analysis: an organizational framework of provenance types and purposes. IEEE Transactions on Visualization and Computer Graphics, 22(1), 31-40.

Ramakrishnan, N., Kumar, D., Mishra, B., Potts, M., \& Helm, RF. (2004). Turning cartwheels: An alternating algorithm for mining redescriptions. In Proceedings of the 10Th ACM SIGKDD international conference on knowledge discovery and data mining, ACM, New York, NY, USA, KDD 2004, pp. 266-275.

Tatusov, R. L., Koonin, E. V., \& Lipman, D. J. (1997). A genomic perspective on protein families. Science, 278(5338), 631-637. https://doi.org/10.1126/science.278.5338.631.

Tatusov, R. L., Galperin, M. Y., Natale, D. A., \& Koonin, E. V. (2000). The cog database: A tool for genomescale analysis of protein functions and evolution. Nucleic Acids Research, 28(1), 33-36.

UNCTAD (2014.) Unctad database. http://unctadstat.unctad.org/

Van Leeuwen, M. (2014). Interactive data exploration using pattern mining. In Interactive knowledge discovery and data mining in biomedical informatics, Berlin: Springer, pp. 169-182.

Walsby, A. (1994). Gas vesicles. Microbiological reviews, 58(1), 94-144.

Webb, G. I. (1996). Integrating machine learning with knowledge acquisition through direct interaction with domain experts. Knowledge-Based Systems, 9(4), 253-266.

Wehrens, R., \& Buydens, L. M. C. (2007). Self and super-organising maps in R: the kohonen package. J Stat Softw, 21(5), 1-19.

WorldBank. (2014). World Bank. http://data.worldbank.org/

Xin, D., Han, J., Yan, X., \& Cheng, H. (2005) Mining compressed frequent-pattern sets. In Proceedings of the 31 st international conference on very large data bases, VLDB endowment, VLDB '05, pp. 709-720.

Yen, J. (1971). Finding the k shortest loopless paths in a network. Management Science, 17, 712-716.

Yen, J. (1972). Another algorithm for finding the k shortest loopless network paths. In Proceedings of 41 st Mtg Operations Research Society of America 20.

Zaki, MJ., \& Phoophakdee, B. (2003). MIRAGE: A framework for mining, exploring and visualizing minimal association rules. Tech. Rep. 03-4, Computer Science Department, Rensselaer Polytechnic Institute.

Zaki, MJ., \& Ramakrishnan, N. (2005). Reasoning about sets using redescription mining. In Proceedings of the 11th ACM SIGKDD international conference on knowledge discovery in data mining, ACM, New York, USA, KDD 2005, pp. 364-373.

Zinchenko, T. (2014). Redescription mining over non-binary data sets using decision trees. Master's thesis, Universität des Saarlandes Saarbrücken, Germany.

Publisher's Note Springer Nature remains neutral with regard to jurisdictional claims in published maps and institutional affiliations. 